\title{
Techniques et interprétation musicales
}

Contenus et stratégies chez les enseignants débutants en didactique de l'instrument

Musical techniques and interpretation: content and strategies for beginning teachers in the didactics of instruments

René Rickenmann, Francisco Márquez Cuesta et Cristina Bellu

\section{OpenEdition}

Journals

Édition électronique

URL : https://journals.openedition.org/educationdidactique/5020

DOI : 10.4000/educationdidactique.5020

ISSN : $2111-4838$

\section{Éditeur}

Presses universitaires de Rennes

\section{Édition imprimée}

Date de publication : 30 décembre 2019

Pagination : $35-57$

ISBN : 978-2-7535-8041-1

ISSN : 1956-3485

\section{Référence électronique}

René Rickenmann, Francisco Márquez Cuesta et Cristina Bellu, « Techniques et interprétation musicales », Éducation et didactique [En ligne], 13-3 | 2019, mis en ligne le 03 janvier 2021, consulté le 11 octobre 2022. URL : http://journals.openedition.org/educationdidactique/5020 ; DOI : https:// doi.org/10.4000/educationdidactique.5020 


\title{
TECHNIQUES ET INTERPRÉTATION MUSICALES CONTENUS ET STRATÉGIES CHEZ LES ENSEIGNANTS DÉBUTANTS EN DIDACTIQUE DE L'INSTRUMENT
}

\author{
René Rickenmann \\ Chercheur associé au Laboratoire de «Didactique des Arts et du mouvement» \\ (DAM), membre de l'équipe de recherche "Educación artística», \\ Universidad Pedagógica Nacional, Colombie \\ Francisco Márquez Cuesta \\ Chargé d'enseignement en didactique des Arts Plastiques et Visuels, \\ "Laboratoire de Didactique des Arts et du Mouvement » (DAM), Université de Genève \\ Cristina Bellu \\ Doctorante et collaboratrice scientifique, \\ "Laboratoire de Didactique des Arts et du Mouvement » (DAM), Université de Genève
}

\begin{abstract}
Enseigner un instrument de musique, notamment pour des enseignants débutants, implique un découpage entre contenus techniques et contenus interprétatifs. L'organisation des leçons en termes de contenus et d'activités peut varier en fonction de la conception de l'enseignant sur l'articulation entre technique et interprétation. Cinq leçons de trois jeunes enseignants en formation ont été observées pendant le deuxième semestre d'une année scolaire. Le geste professionnel de définition a été analysé avec les méthodes quantitative et qualitative. Deux cas (1 et 3) s'inscrivent dans une conception de technique puis d'interprétation, alors qu'un autre (cas 2) relève d'une articulation équilibrée entre technique et interprétation. Bien que pour les trois cas l'empreinte personnelle soit restée constante, l'on constate des différences importantes concernant la construction d'un objet enseigné plus complexe pour le cas $2^{1}$.
\end{abstract}

Mots-clés : technique, interprétation, professionnalisation, gestes professionnels de définition, formation des enseignants.

Musical techniques and interpretation: content and strategies for beginning teachers in the didactics of instruments

Teaching a musical instrument, especially to beginners, implies a scission between technical and interpretative content. The organization of lessons in terms of activities and content may vary following the conception the teacher has about the articulation of technique and interpretation. Five lessons of three young teachers were observed during the second semester of a school year. The professional gesture of definition was analysed with both quantitative and qualitative methods. The first case followed a conception of technique before interpretation, whereas the two others used an equal articulation of technique and interpretation. Although their personal imprinting remained constant, a sign of professionalization can be seen in less use of definition and more of other professional gestures, such as institutionalization, devolution, and regulation and a better articulation between technique and interpretation during lessons ${ }^{2}$.

Keywords : technique, interpretation, professionalization, professional gestures of definition, beginning teachers. 


\section{QUELQUES REPÈRES AUTOUR DES QUESTIONS SUR L'INTERPRÉTATION MUSICALE}

L'interprétation musicale reste aujourd'hui une question vive qui concerne très directement les processus de formation des musiciens instrumentistes et chanteurs. Cette question a été abordée à partir de divers champs de connaissance : celui de l'analyse des pratiques liées à l'écoute puis à la performance musicale elle-même, en passant par différents domaines d'études de la musique tels que la musicologie, l'histoire de la musique, la critique ou la composition. À leur tour, ces travaux ont fait d'importants emprunts à divers domaines des sciences humaines comme l'histoire, l'ethnologie, la psychologie, la sémiotique ou la sociologie, pour ne nommer que les plus visibles. Parmi ces domaines, on peut constater qu'il y a peu de recherches menées sur la question de l'enseignement de l'interprétation, notamment auprès des débutants, que ce soit dans le cadre des sciences de l'éducation ou dans celui, en particulier, de la didactique de la musique. Cela malgré le fait que ces recherches peuvent sans doute apporter de nouveaux éclairages sur la question de l'interprétation, en lien avec les problématiques spécifiquement didactiques qui se posent à l'enseignant.

Dans ce texte, nous souhaitons apporter quelques éléments de réflexion sur l'enseignement de l'interprétation instrumentale, issus de l'analyse d'une partie du corpus d'une recherche mixte réalisée auprès des enseignants en formation, de leurs formateurs en didactiques et de certains de leurs élèves, et plus particulièrement au travers des dispositifs de pratiques pédagogiques des premiers ${ }^{3}$. Cette analyse part de deux axes de problématisation : les principales questions liées aux conceptions de l'interprétation du musicien instrumentiste s'enchâssent ici avec celles didactiques liées à l'identification, le découpage, l'ordonnancement et l'articulation de contenus visant la construction, par les élèves, de savoirs sur l'interprétation instrumentale. Dans cet aparté, nous abordons trois ensembles de problématiques liées à la question de l'interprétation qui sont particulièrement en rapport avec des questions didactiques relevées dans l'enseignement instrumental, notamment auprès d'élèves débutants.

Un premier ensemble aborde la question centrale du rôle du musicien instrumentiste comme médiateur d'un répertoire d'œuvres. Étudié notamment par l'ethnomusicologie, cet ensemble concerne la question de la tension entre les deux régimes de transmission du répertoire que sont l'oral et l'écrit (Da Lage \& Vandiedonck, 2001) en termes de fidélité à l'œuvre, à la sonorité d'une époque donnée (Harnoncourt, 1982), aux significations que l'on prête historiquement aux termes d'exécutant et d'interprète pour qualifier le musicien instrumentiste (Willener, 1997) et son rapport aux intentions expressives du compositeur. Cela concerne notamment le répertoire classique occidental car c'est à mesure que l'activité de l'interprète se détache de celle du compositeur, que leurs techniques gagnent en spécificité, la part de liberté qui revient à l'interprète s'amenuise et celui-ci devient de plus en plus soumis au texte écrit $»$ (Bosseur, 2005, p. 76-77). Cet ensemble concerne donc ce qu'Imberty désigne comme tensions entre l'objectif et le subjectif dans le rapport à la musique, dont il refuse le découplage radical en plaçant l'interprétation comme un objet tiers, dynamique et situé, produit de la co-définition du système musicien-œuvre :

... dans le domaine des objets culturels, la représentation interne que le sujet se forge de l'objet (représentation qui est par nature mouvante et changeante) peut aller jusqu'à modifier la structure de l'objet lui-même lorsque celle-ci est médiatisée par l'action du sujet (une exécution par exemple) (Imberty, 1998, p. 218).

Un deuxième ensemble de questions concerne le rapport à la partition comme trace de l'œuvre. La tendance majoritaire à stabiliser la notation musicale, initiée en Europe au $18^{\mathrm{e}}$ siècle et ayant eu son apogée au $19^{e}$ siècle, renforce le besoin d'arriver au « sens » de l'œuvre 4 à travers une approche de type herméneutique (Arbo, 2000) du fait que:

[...] la notation musicale n'est en rien identique au langage musical [...] Le signe musical n'est qu'une instruction donnée à l'exécutant pour qu'il produise par le moyen de sa voix ou de son instrument un son avec une hauteur et une durée particulières, tout en ajoutant, à certaines époques historiques, des suggestions concernant le rythme, la dynamique et l'expression, ou des indications sur le rapport avec d'autres sons (par le moyen de la liaison, du coulé, etc.). Tous ces éléments de la matière tonale ne peuvent être qu'approximativement ordonnés, et la charge d'obtenir 
les effets indiqués est laissée à l'exécutant. (Schütz, 2006, p. 19. Nous soulignons).

La perspective sémiotique développée autour des années 1970-1980 complexifie par ailleurs les rapports qu'entretient le musicien avec les différentes dimensions de l'œuvre. Ainsi, dans une approche qui fait désormais référence, Nattiez (1987) souligne l'épaisseur de l'œuvre comme résultat de l'interaction des trois dimensions, "esthésique » (réception historique de l'œuvre), «poiétique » (processus de sa composition/production) et "neutre » (sa forme et fonctionnement en tant que langage musical). Dans le cadre d'autres paradigmes, certaines études sur les stratégies de travail des instrumentistes experts (Dogantan-Dack, 2012; Héroux \& Fortier, 2014) font écho à cette complexification sémio-pragmatique du rapport musicien-œuvre via la partition, en analysant l'interprétation comme processus émergeant. Des travaux récents (Hastings, 2011), par exemple, définissent l'interprétation comme un processus macro-micro-macro orientant la lecture de la partition selon trois types de caractéristiques : fixes (tons ou hauteurs, rythmes), variables (notations liées à l'expressivité) et implicites (structures, informations historiques et contextuelles). En ce sens, le travail d'interprétation est considéré comme un processus de reconstruction de l'œuvre à partir des traces écrites : " first they gain a conception of the composition as a whole, then they work out technical and interpretive problems, then they integrate the parts back into the whole " (Hasting, 2011, p. 370).

L'attention aux situations d'enseignement impliquant des débutants convoque un troisième ensemble de questions relatives aux rapports de l'apprenti musicien avec l'instrument. Les musiciens s'accordent pour dire que de nombreux aspects du jeu interprétatif échappent largement aux écritures et que l'interprétation musicale se joue essentiellement dans la performance comme processus qui ... signifie bien cet aspect incarné (par des êtres et des corps humains) du son, du temps musical pris dans une temporalité située» (Ravet, 2005, p. 11. Nous soulignons). Ces questions concernent des aspects organologiques (Cahiers d'ethnomusicologie, 1989; Boissière, 2011; Sève, 2011) et ergonomiques (Baily, 2001) saisis dans leurs aspects technico-culturels, dans le sens des « techniques du corps» de Mauss (1934). Certains travaux abordent aussi les aspects ontologiques du geste musical (Blum, 2001) et de
« l'origine corporelle de l'instrument de musique » au sens de Schaeffner (Boissière, 2011). Ces questions touchent autant ce «qu'il y a à faire » que ce « qu'il y a à entendre " en vue de construire la base sonore des œuvres jouées à partir d'un système corps-instrument (Rickenmann \& De la Calle, 2015).

\section{DES QUESTIONS DIDACTIQUES AU CEUR DE L'ENSEIGNEMENT DE L'INTERPRÉTATION}

Dans une perspective descriptive du « didactique ordinaire » (Schubauer-Leoni \& Leutenegger, 2002), l'étude de l'enseignement des savoirs concernant l'interprétation peut apporter des éclairages sur la transmission et les transformations historiques des œuvres et des pratiques musicales et culturelles. En effet, les problématiques évoquées comportent, certes, des aspects « descendants » liés à l'épaisseur historique et culturelle des savoirs et pratiques autour de cette notion, mais aussi des éléments concernant les aspects émergents et dynamiques. Par exemple, dans une perspective de cognition située sur la construction experte du projet musical (Goasdoué, 2004), des travaux considèrent l'interprétation comme émergence d'un ensemble de contraintes imposées par la partition, l'instrument et les capacités du musicien. Ces recherches nous permettent de faire le lien avec une perspective d'analyse de l'enseignement, en termes d'habilitations et de contraintes qui se posent à l'apprenant dans l'interaction avec les milieux didactiques définis par les enseignants.

Dans cet ordre d'idées, ces différents aspects conduisent vers deux questions centrales pour notre étude : celle des rapports de l'élève aux deux milieux didactiques que sont l'instrument et l'œuvre et celle des contenus que les enseignants font circuler pour définir et orienter ces rapports.

\section{Les deux topoï des savoirs interprétatifs}

Dès lors que l'apprentissage de l'interprétation par des élèves débutants cible le développement d'une expertise d'action par rapport aux deux milieux didactiques mentionnés, une première problématisation didactique de notre recherche concerne la description des stratégies enseignantes visant la production des topoï (Sensevy \& Mercier, 2007) relatifs à ces deux milieux, autrement dit les postures et 
les conduites de l'élève qui lui sont nécessaires pour « évoluer dans» et « les faire évoluer».

Un premier topos concerne les relations entre le musicien interprète et l'œuvre musicale, que la recherche sur les savoirs d'experts situe notamment via l'approche herméneutique (Arbo, 2000) et pragmatique à la partition (Dogantan-Dack, 2012, Hastings, 2011) comme moyen d'accès à l'œuvre. Un autre lien, thématisé dans les pratiques musicales notamment à partir du $20^{\mathrm{e}}$ siècle, se situe du côté du rapport sensible à la musique (Imberty, 1988 ; 1997) de ce musicien interprète, mais aussi le rapport sensible à son instrument (Goasdoué, 2004). Concernant ce premier topos, les travaux d'Imberty (1988) montrent que l'œuvre est par nature "malléable et labile » et ne se réalise que dans le rapport performatif ${ }^{5}$. Pour l'enseignant se pose alors la question des stratégies d'accès à l'œuvre qui, dans l'enseignement ordinaire, passent principalement par des savoirs concernant la lecture des partitions, en particulier l'usage des écritures-indices adressées au musicien instrumentiste concernant le jeu instrumental. Ce travail relève d'une approche herméneutique en ce que la construction du sens des paramètres sonores de l'œuvre implique des savoirs contextuels qui ne sont pas conventionnellement « contenus » dans les signes utilisés ${ }^{6}$. Bien qu'un certain nombre de ces savoirs contextuels puisse faire l'objet de « mises en textes», ils sont le plus souvent communiqués à travers les modalités de la transmission orale et par monstrations au sein des écoles de musique, des conservatoires ou dans le cadre de la préparation de concerts. La problématisation didactique de ce topos auprès des débutants implique donc un travail de nature technique-solfégique lié au décodage des partitions. Mais, les enseignants vont aussi au-delà de cet aspect technique afin de permettre l'accès aux caractéristiques variables et implicites de l'œuvre, pour reprendre le modèle de Hastings. À partir de stratégies d'analyse formelle, dans l'alternance de modalités de lecture de la partition et d'écoute des productions sonores de l'enseignant, ils visent la mise en évidence d'aspects structuraux saillants de l'œuvre et d'éléments, notamment esthésiques (Nattiez, 1987), qui dévoilent l'épaisseur historique de l'œuvre.

Un second topos concerne le rapport musicieninstrument orienté par la nature performative de la musique. Dans les domaines artistiques, la performance s'appuie le plus souvent sur une corporéité spécialisée nécessitant un long travail de ruptures avec le corps quotidien via des préparations, des exercices et des entrainements qui en visent le développement et le maintien à un haut niveau. La problématisation didactique de ce topos implique, cependant, que ces modalités ne se réduisent pas seulement à une approche technique concernant la sensibilité physiologique et la production mécanique du geste. En effet, la nature incarnée et située de la production instrumentale implique que l'enseignant contribue à la mise en place de ce que Blum (2001) appelle un « geste des gestes », concernant la sensibilité du musicien interprète. C'est en ce sens que d'aucuns soulignent, par exemple, pour le chant, la nécessité de « combattre l'illusion de la naturalité des compétences et de la fixité de l'œuvre » (Gonzalez Martinez, 2000, p. 282 cité par Blum 2001). Cette sensibilité étant, certes, liée aux œuvres jouées par l'instrumentiste, mais aussi aux spécificités du système corps-instrument qui fournit la base sonore des œuvres jouées (Rickenmann \& De la Calle, op. cit.).

\section{Trois conceptions d'enseignement comme outils didactiques}

Le développement des topoï d'expertise suppose également celui des savoirs spécialisés permettant de les exercer. Compte tenu des trois ensembles de questions mentionnés dans la première partie concernant l'interprétation, l'enseignement d'un instrument de musique, notamment à ses débuts, implique de la part de l'enseignant qu'il procède à discriminer différents types de contenus à enseigner permettant à l'élève d'habiter les deux topoï, notamment :

- ceux relatifs à la maîtrise de l'instrument, qui sont habituellement considérés comme relevant de la technique instrumentale; - puis, dans une continuité dont la frontière est très poreuse, ceux concernant la musique qui peut être produite avec cet instrument, avec la prise en compte d'une dimension interprétative impliquant la sensibilité du musicien;

- ceux en lien avec l'apprentissage de la notation musicale qui comporte des enjeux techniques relatifs au décodage tout comme des enjeux interprétatifs en lien avec l'analyse et la compréhension de l'œuvre. Cela implique des connaissances historiques et contex- 
tuelles qui ne figurent pas explicitement dans la partition;

- ceux qui relèvent des gestes d'étude hors-leçon, assurant l'entraînement et la maîtrise des savoirs qui circulent durant les leçons.

Une constante dans les problématiques évoquées est l'organisation de ces différents contenus, sous la conjonction entre une dimension technique visant la maîtrise de l'instrument ainsi que les règles de la notation musicale, et une dimension interprétative visant la construction de l'épaisseur de l'œuvre à partir de la partition ainsi que le développement d'un rapport sensible à la sonorité instrumentale.

Par rapport à la gestion enseignante de cette articulation, se dégagent trois conceptions. Une première, la plus répandue, est celle qui considère les savoirs techniques comme un corpus à caractère universel et général préalable à l'interprétation musicale. Dans les processus de didactisation liés à cette conception, l'enseignement pose comme préalable un minimum de maîtrise technique qui semble nécessaire avant d'aborder des aspects de type interprétatif (conception « technique PUIS interprétation »). À l'opposé, une conception naturaliste de la « musicalité » qui serait potentiellement présente dans tout individu et dont les aspects techniques ne seraient nécessaires qu'à son accomplissement (conception « interprétation PUIS techniques de base »). Cette conception est bien moins fréquente dans l'enseignement musical, mais présente dans les activités musicales scolaires et extra-scolaires auprès de jeunes élèves. Par exemple, sous la forme d'activités d'improvisation spontanée avec des instruments musicaux, mais qui font l'impasse sur des orientations techniques/musicales particulières. Une troisième conception, finalement, consiste en l'articulation permanente des apports techniques aux apports interprétatifs en fonction des répertoires d'œuvres. Dans cette troisième conception, le recours aux techniques instrumentales tient davantage de la généricité que de la généralité : en référence à la notion de genre en littérature (Rastier, 2001), les savoirs techniques feraient ici référence à des répertoires techniques dont l'organisateur serait les œuvres musicales appartenant à des genres ou à des styles... et donc, avec la prise en compte d'aspects hétérogènes, cependant réunis ou assemblés dans le «tout » des répertoires d'œuvres et de pratiques musicales spécifiques et différenciées (conception «interprétation ET techniques afférentes »).

\author{
Larticulation " technique - interprétation » \\ et les modalités de définition des milieux \\ et des savoirs didactiques
}

L'enseignement de l'interprétation auprès des musiciens instrumentistes, notamment ceux débutants, semble donc concerner deux ordres de questions didactiques auxquels sont confrontés les enseignants. D'une part, celui de la gestion de l'équilibre entre les dimensions techniques et interprétatives concernant la définition des savoirs relatifs tant au rapport à l'œuvre musicale qu'à celui musicieninstrument. D'autre part, celui du travail enseignant sur les modalités d'accès à ces savoirs et ces deux topoï, étant donné les caractéristiques a) de savoirs incorporés et situés pour le premier et b) le caractère émergeant et souvent largement implicite de l'œuvre pour le second (concernant les leçons observées).

Relativement au premier ordre de questions, dans l'apprentissage d'instruments de musique, l'une des tendances marquées depuis la fin du $19^{e}$ siècle a été celle de développer un apprentissage méthodique et progressif lié, notamment, à la stabilisation du système de notation musicale. Ce processus a donné lieu à différentes méthodes d'instrument proposant aux enseignants une organisation théorique des notions et savoirs travaillés, ainsi qu'une organisation pratique et séquencée d'exercices, d'études et de pièces musicales organisées, pouvant s'inscrire dans l'une ou l'autre de ces conceptions de la progression des apprentissages. Les recherches conduites par l'un des auteurs de ce texte ${ }^{7}$ sur les méthodes d'enseignement du violoncelle montrent, concernant la problématique des rapports entre savoirs techniques et savoirs interprétatifs dans l'enseignement instrumental, qu'elles adoptent le plus souvent une tendance très majoritairement orientée vers une conception «technique PUIS interprétation». Dans cette organisation didactique, l'œuvre musicale et des aspects liés à l'interprétation, interagissent avec l'enseignement de techniques instrumentales. Cela à travers des pièces organisées par ordre de difficultés qui sont intercalées avec les exercices portant sur des aspects spécifiques de la technique instrumentale. La prépondérance de cette conception relevée par cette étude, nous amène à formuler deux remarques importantes pour notre analyse. D'abord, le fait que majoritairement les enseignants ne se calent pas de manière rigide sur une méthode spécifique, ayant plutôt tendance à diversifier l'usage de ressources 
pédagogiques en provenance de méthodes et publications variées. Cela nous invite à la prudence, en termes d'analyse des praxéologies observées, quant à l'usage des ressources pédagogiques de ces enseignants pour identifier la mobilisation de conceptions prépondérantes par rapport aux contenus techniques et interprétatifs dans les leçons. Ensuite, indépendamment des dispositions particulières de chacun des enseignants concernant les référents et conceptions de l'enseignement, il faut souligner que les formes scolaires mobilisées ont également un poids non négligeable sur leurs choix didactiques (Vincent, 1996). Ainsi, la structure tripartite « échauffement / exercices / jeu musical » qui s'est généralisée pour les enseignements des disciplines impliquant la corporéité (en danse, théâtre, musique, éducation physique), tant au niveau de la leçon que sur l'empan plus large de l'année scolaire, constitue un important organisateur ${ }^{8}$ qui contraint les choix et fonctionnements didactiques des enseignants en lien avec l'une ou l'autre de ces conceptions. L'on peut donc constater que l'enseignant d'instrument ou du chant, faisant nécessairement face à des facteurs internes à la situation didactique, est contraint dans certains de ses choix par des facteurs externes, telle la conception des rapports technique versus interprétation ou les structures qui organisent institutionnellement l'enseignement.

Concernant le second ordre de questions, de par la nature des savoirs en jeu, le caractère conjoint de l'action didactique (Sensevy \& Mercier, 2007) contraint aussi l'enseignant à faire des choix quant à la manière de choisir un milieu didactique et d'y introduire les contenus à l'intention de l'élève : définir certaines tâches telles que répéter un exercice ou faire une improvisation, faire des ostensions en soulignant une production sonore de l'élève ou en montrant la partition, faire des démonstrations à imiter, etc. Ces choix constituent des approches diversifiées dont la pertinence dépend aussi de la sensibilité de l'enseignant au contexte et à la conjoncture de la leçon. Dès lors que l'enseignement de l'interprétation implique des savoirs incorporés et situés et que l'approche à l'œuvre concerne des aspects émergeants et implicites, le choix des modalités de définition des milieux et des savoirs en jeu semble constituer un enjeu majeur de l'action enseignante.

\section{MÉTHOdOLOGIE ET CORPUS}

Les rapports entre l'enseignement des techniques et l'interprétation musicale permettent de saisir, d'une part, les défis spécifiques que posent les interactions entre les savoirs et les pratiques disciplinaires de référence; d'autre part, les dispositifs d'enseignement et leurs effets. Les analyses présentées dans ce texte font partie d'une étude plus vaste s'inscrivant dans une méthodologie de type mixte (analyse quantitative et analyse qualitative), concernant les dispositifs de formation didactique pour les futurs enseignants en musique de l'instrument ou du chant, dans plusieurs Hautes écoles de musique en Suisse9, dont nous avons sélectionné trois cas présentant des spécificités remarquables concernant notre problématique.

Le fait que pour le corpus retenu nous soyons face à des enseignants en formation initiale ( $1^{\text {re }}$ année pour Violoncelle $1 \mathrm{~A}$ et $2^{\mathrm{e}}$ année pour les deux autres cas), nous incite également à prendre en compte, dans l'analyse, des données indicatives concernant les processus de progression de ces enseignants en formation dans le cadre de leurs pratiques. Les caractéristiques de ce corpus nous permettent de contraster les analyses sur la question de l'enseignement de l'interprétation avec les indicateurs de progression, ces derniers venant ainsi nuancer certaines observations.

\section{Catégorisation et données quantitatives}

Le corpus des données s'articule autour de 5 leçons dispensées par chaque enseignant en formation à son élève (de L0 à L4). Pour cette étude, nous avons retenu trois cas: deux séries de leçons de violoncelle et une série de leçons de harpe. Ces cas présentent la caractéristique commune de se rapporter à des élèves débutants de l'instrument. Les cas Violoncelle 1A (concernant l'enseignant en formation Ste) et Harpe 1A (concernant l'enseignante en formation Sti) s'adressent à des débutants adultes. Pour le cas Violoncelle 1B (concernant l'enseignant en formation Sth) il s'agit d'un débutant beaucoup plus jeune, neuf ans environ, à l'exception de L3 durant laquelle l'enseignant en formation a donné sa leçon à une élève avancée. La première leçon (LO) nous sert à donner un profil de l'enseignement de 
chaque enseignant en formation afin d'éclairer l'analyse qualitative.

En fonction des problématiques, nous suivons les modèles de l'action enseignante proposés et développés par la Théorie de l'action conjointe didactique (Sensevy \& Mercier, 2007), notamment à la suite de la Théorie des Situations didactiques de Brousseau (1991). Rappelons que selon ce modèle, l'enseignement peut être décrit comme un aménagement de situations dans lesquelles des jeux d'apprentissage invitent l'élève à interagir avec un milieu didactique. Les adaptations de l'élève durant les jeux produisent des apprentissages. "S'il veut faire jouer le jeu didactique, le professeur doit faire jouer des jeux d'apprentissage. Pour cela, il doit satisfaire à quatre conditions » (Sensevy, 2006, p. 201) : l'enseignant doit « définir le jeu » afin que l'élève joue le bon jeu; par ailleurs, il doit réussir à " dévoluer » pour que l'élève se fasse responsable et accepte de se confronter aux enjeux de la situation; en fonction de la manière dont l'élève agit face à ces enjeux, s'il s'avère nécessaire, l'enseignant doit également « réguler » les interactions élève-milieu afin que celui-ci puisse jouer le jeu et avancer; finalement, l'enseignant doit satisfaire à la condition « d'institutionnalisation » qui consiste à signaler à l'élève la dimension culturelle et partagée des connaissances qu'il a produites durant son jeu.

Dans la mesure où l'analyse statistique implique que soient générées assez rapidement un nombre important de données, nous avons seulement catégorisé les « actions de définition » à travers lesquelles l'enseignant définit les éléments du jeu didactique (Sensevy, Mercier, Schubauer-Leoni, 2000; Sensevy, 2006) :

La condition de définition du jeu réfère à une autre nécessité : le collectif didactique doit pouvoir jouer sans ambiguité le bon jeu, celui dont le professeur sait qu'il va amener les élèves à avancer. On pourra donc étudier comment les règles définitoires du jeu sont transmises par le professeur et appropriées par les élèves, jusqu'à une certaine « transparence » qui permette de jouer le bon jeu presque « sans y penser» (Sensevy, 2006, p. 210. Nous soulignons).

Nous avons procédé à un codage à travers la catégorisation de 6 modalités enseignantes de définition du jeu didactique : a) des tâches, b) discursives, c) d'exemples ou d) de contre-exemples, e) des ostensions et pointages, puis f) des métaphores; d'autre part, nous avons également codé une série de 4 catégories de savoirs à enseigner convoqués via ces actions de définition des enseignants en formation. Ces sont les données obtenues concernant cette deuxième série que nous analysons principalement dans ce texte:

- les savoirs techniques concernant l'instrument, le rapport corps-instrument et les aspects solfégiques (MT). Ces savoirs concernent la lecture de partitions mais ne s'y réduisent pas,

- d'autres savoirs impliquant un rapport d'appropriation qui dépasse le décodage de la notation musicale et donnent l'accès à la lecture de partitions et à l'écriture de l'œuvre musicale (ML),

- les savoirs relevant de l'interprétation de l'œuvre et de la posture esthétique de l'interprète (MI),

- les savoirs relatifs à l'organisation des études en dehors des moments de la leçon (MH).

L'ensemble des codages a été réalisé en double aveugle, avec une stabilisation ultérieure des données codées sous forme de verbatims, comme le montre l'exemple suivant :

\begin{tabular}{|c|c|c|}
\hline Temps & $\begin{array}{l}\text { Définition / } \\
\text { Savoirs / } \\
\text { Agent }\end{array}$ & Transcription \\
\hline 04.14 & Dt/ MLMI/Sti & $\begin{array}{l}\text { STi : Je vais jouer la mélodie } \\
\text { et on va déterminer le phrasé. } \\
\text { Indique-moi chaque fin de } \\
\text { phrase: } \\
\text { Lorsque c'est un petit bout ou } \\
\text { plusieurs petits, à l'intérieur } \\
\text { d'un grand. }\end{array}$ \\
\hline 05.48 & Dp/ ML/Sti & $\begin{array}{l}\text { Donc il y a une première fin qui } \\
\text { se trouve ici (pointe et souligne } \\
\text { la partition). Tu es à cheval sur } \\
\text { la fin de cette grande phrase- } \\
\text { là et le début de la suivante. } \\
\text { (pointe la partition). }\end{array}$ \\
\hline
\end{tabular}

Dans cet exemple, les deux expressions soulignées en gras indiquent la tâche (Dt) que l'enseignante Sti donne à son élève à ce moment-là de la leçon; celles soulignées indiquent les savoirs en jeu : l'expression "le phrasé" indique une orientation vers des savoirs interprétatifs (MI) qui concernent un niveau de lecture de la partition qui va au-delà du décodage solfégique (« indique-moi chaque fin de phrase »). Du point de vue du traitement quantitatif des données, cette manière de faire nous permet 
de coder les occurrences pour chaque mode de définition utilisé, puis celles des savoirs en jeu dans les différents moments de la leçon. Il faut souligner que tant que la modalité ou les savoirs en jeu ne changent pas dans la leçon, nous ne comptons que la première manifestation de l'occurrence. Par contre, lorsque la modalité de définition change, même s'il s'agit toujours du "même" savoir, nous comptons comme une nouvelle occurrence tant en ce qui concerne la modalité (en 05:48 un pointage) qu'en rapport au savoir (ici, pour le codage ML, une orientation sur des caractéristiques de la partition).

Dans ce texte, nous partons d'analyses descriptives de chaque cas, basées sur ces données quantitatives, qui nous permettent de poser des hypothèses de travail orientant leur analyse qualitative. Pour réaliser cette dernière, nous avons recours aux synopsis des leçons, réalisés à partir des codages effectués pour la partie quantitative, et procédons au choix d'événements remarquables concernant des phénomènes didactiques identifiés. Pour ces événements remarquables identifiés au niveau intra-leçon, nous opérons des transcriptions plus fines concernant les actions et verbalisations des acteurs, puis analysons également leur évolution inter-leçons. Nous cherchons ainsi à décrire et à comprendre les logiques locales (intra-leçon) de l'agir enseignant, que nous contrastons en permanence avec celles qui se dégagent globalement de l'ensemble des 5 leçons observées. En fonction de la problématique évoquée nous orientons nos analyses sur deux questions de recherche : quels contenus, et leurs articulations, sontils privilégiés par ces différents enseignants en formation, et comment sont-ils organisés dans le continuum intra et inter-leçons? Quels sont les effets sur les deux topoï musicien-instrument et musicien-œuvre?

Dans la suite de ce texte nous présentons des résultats d'analyse pour ces deux questions, en soulignant des spécificités pour chacun des trois cas, dans l'ordre: Violoncelle 1A, Harpe 1A et Violoncelle 1B.

\section{Gestion de l'articulation technique/ interprétation, organisation didactique des savoirs abordés et profil des enseignements}

La figure 1 montre les histogrammes 1 (Violoncelle 1A), 2 (Harpe 1A) et 3 (Violoncelle 1B) concernant les leçons de référence (LO) des trois enseignants en formation. Ces histogrammes nous donnent des indications10 sur la manière dont ils abordent les articulations ou les équilibres entre savoirs techniques (MT) et savoirs interprétatifs (MI) à ce moment de l'année (décembre), et également les rapports avec les deux autres types de savoirs qui ont été codés, le travail à la maison (MH) et la lecture de partition (ML).

Pour les chercheurs habitués à l'analyse de situations didactiques de l'enseignement scolaire ordinaire, la distribution d'actions de définition enseignante que présentent ces histogrammes est à première vue surprenante : l'enseignant d'instrument semble être en train de définir « en permanence »! En effet, les histogrammes montrent une distribution de définitions de savoirs tout au long des leçons, notamment ceux de nature technique, ce qui semble être un trait caractéristique des leçons d'instrument données auprès de débutants ${ }^{11}$. Cette caractéristique traduit une spécificité de la dimension conjointe de l'action didactique pour ce type d'enseignement, qui relève de la dimension incarnée du topos musicieninstrument : l'enseignant ne peut définir le milieu et les tâches qu'à partir des apports de l'élève, en particulier la production sonore. Cette dernière constitue, in fine, le matériau principal avec lequel l'enseignant définit un milieu de travail pour l'élève : or, comme ce matériau est très labile et demande des ajustements permanents, cela se traduit par un découpage important des définitions de savoirs en jeu sur l'ensemble du temps de la leçon. En effet, auprès des élèves débutants, ces ajustements prennent souvent un caractère " terme à terme », qui met en avant la dimension conjointe et conduit l'action enseignante de (co-)définition à ce découpage prononcé. Sur la base des données quantitatives, l'analyse des synopsis démontre qu'en termes de dévolution et de responsabilité devant la tâche, la production de ce matériau didactique relève davantage de l'action de définition que de celle de régulation.

Dans la figure 1 , les histogrammes 1 à 3 permettent de constater que, dans les trois leçons de référence (LO), il y a un nombre assez important de MT par rapport aux MI, et qu'ils sont par ailleurs distribués sur l'ensemble de chacune des leçons. La moyenne des occurrences pour l'ensemble des leçons figurant dans les tableaux 1, 2 et 3, qui présentent les occurrences (en pourcents) pour chaque savoir défini durant l'ensemble des leçons, confirme l'orientation de ces enseignements sur des aspects techniques. Lorsque l'on observe, pour les deux cas concernant le violoncelle, 
Figure 1

Histogramme a : Violoncelle $\mathrm{I}_{\mathrm{A}}$ (Ste)-Distribution des savoirs abordés dans LO.

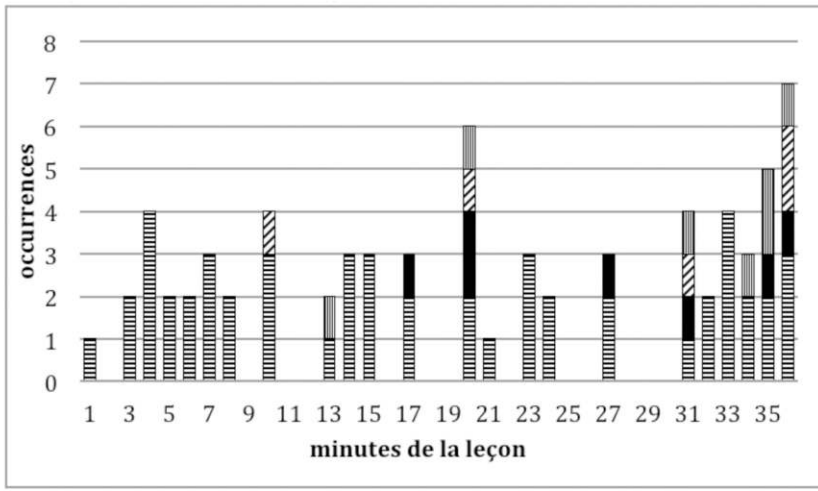

Histogramme b : Violoncelle $\mathrm{I}_{\mathrm{A}}$ (Sti)-Distribution des savoirs abordés dans LO.

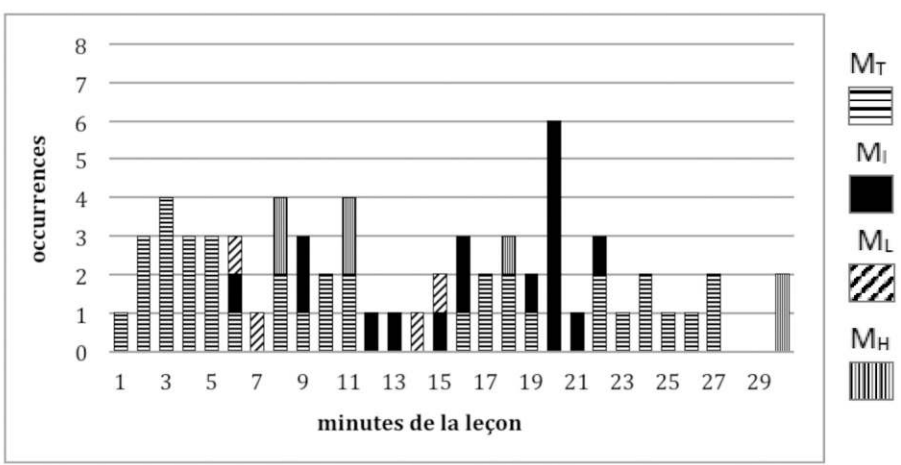

Histogramme c : Violoncelle $\mathrm{I}_{\mathrm{B}}$ (Sth)-Distribution des savoirs abordés dans LO.

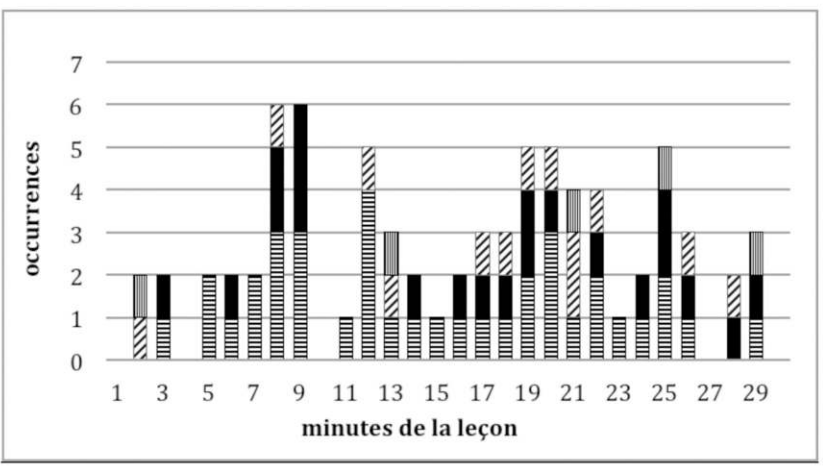

les moyennes de MT et de MI, apparaît une prédominance de MT autour de $60 \%$ contre $20 \%$ d'occurrences $\mathrm{MI}$, chiffres qui suggèrent une tendance à s'inscrire dans la conception « technique PUIS interprétation $»$. Bien que nous ne disposions que d'une leçon auprès d'une élève avancée (L3), les chiffres pour Violoncelle 1B (24\% MT contre $62 \%$ MI) confirment, comme le montrent les analyses qualita- tives, l'adhésion à cette conception. Le cas Harpe 1A contraste avec des pourcentages MT et MI autour de $40 \%$ pour les deux types de savoirs, ce qui indiquerait que les leçons mobilisent plutôt une conception « interprétation ET techniques afférentes ».

Deux autres indices articulés peuvent être mobilisés pour confirmer l'adhésion de ces enseignements à l'une de ces deux conceptions : d'une part, 
le couplage ou l'absence de couplage entre MT ou MI avec les autres savoirs; d'autre part, le moment de la leçon où sont principalement travaillés les savoirs MI. Pour L0, l'on constate que pour les deux cas de violoncelle il y a des savoirs techniques non couplés (surtout chez Violoncelle 1A) et que les MI sont systématiquement couplés avec des MT pour les deux cas. Cela traduirait une permanence des savoirs techniques même lorsque des thématiques liées à l'interprétation des pièces sont en jeu.

Bien qu'avec certaines nuances et une variabilité importante, lorsque l'on observe les histogrammes des autres leçons ( $c f$. figure 2 pour le Violoncelle $1 \mathrm{~A}$ et figure 4 pour le Violoncelle 1B), les données confirment cette lecture. Pour Violoncelle 1A, les MI se concentrent surtout en fin de leçon (sauf dans L3) et sont presque systématiquement couplés avec des MT sauf pour Ll où en fin de leçon une grande partie est dédiée uniquement au jeu interprétatif d'une œuvre; pour Violoncelle 1B, dans les leçons L1, L2 et L4 auprès des débutants, les MI sont également souvent couplés avec des MT, bien qu'ils émergent beaucoup plus tôt dans la leçon. Pour ce cas, L1 constitue aussi une exception car des MI sont travaillés exclusivement vers la fin de la leçon.

Tableau 1 : Violoncelle IA (Ste) :

Savoirs abordés de L0 à L4, en pourcents

\begin{tabular}{|l|l|l|l|l|l|}
\hline Leçons & MT & MI & ML & MH & Occurrences \\
\hline L0 & 73.2 & 9.8 & 7 & 9.8 & 71 \\
\hline L1 & 50 & 38.2 & 0 & 11.7 & 34 \\
\hline L2 & 69.2 & 12.3 & 9.2 & 9.2 & 65 \\
\hline L3 & 57.1 & 28.5 & 6.1 & 8.1 & 49 \\
\hline L4 & 60.6 & 24.2 & 6.0 & 9 & 33 \\
\hline moyenne & $62 \%$ & 22.6 & 5.6 & 9.6 & \\
\hline
\end{tabular}

Tableau 2 : Harpe IA (Sti) :

Savoirs abordés dans L0 à L4, en pourcents

\begin{tabular}{|l|l|l|l|l|l|}
\hline Leçons & MT & MI & ML & MH & Occurrences \\
\hline L0 & 56.9 & 26.1 & 6.1 & 10.7 & 65 \\
\hline L1 & 40 & 38.7 & 17.5 & 3.7 & 80 \\
\hline L2 & 38.2 & 48.9 & 8.5 & 4.2 & 47 \\
\hline L3 & 21.4 & 61.9 & 11.9 & 4.7 & 42 \\
\hline L4 & 44.4 & 35.5 & 11.1 & 8.8 & 45 \\
\hline moyenne & 40.2 & 42.2 & 11 & 6.4 & \\
\hline
\end{tabular}

Tableau 3 : Violoncelle IB (Sth):

Savoirs abordés dans LO à L4, en pourcents

\begin{tabular}{|l|l|l|l|l|l|l|}
\hline Leçons & MT & MI & ML & MH & $\begin{array}{l}\text { Occur- } \\
\text { rences }\end{array}$ & $\begin{array}{l}\text { Niveau } \\
\text { élève }\end{array}$ \\
\hline L0 & 48.6 & 27.6 & 17.1 & 6.5 & 76 & $\begin{array}{l}\text { Débu- } \\
\text { tant }\end{array}$ \\
\hline L1 & 76.1 & 14.2 & 0 & 9.5 & 21 & $\begin{array}{l}\text { Débu- } \\
\text { tant }\end{array}$ \\
\hline L2 & 74.1 & 16.1 & 9.6 & 0 & 31 & $\begin{array}{l}\text { Débu- } \\
\text { tant }\end{array}$ \\
\hline L3 & 23.8 & 61.9 & 9.5 & 4.7 & 21 & Avancé \\
\hline L4 & 58.6 & 17.2 & 10.3 & 13.7 & 28 & $\begin{array}{l}\text { Débu- } \\
\text { tant }\end{array}$ \\
\hline moyenne & 57.9 & 23.8 & 11.1 & 7.1 & & \\
\hline $\begin{array}{l}\text { Moyenne } \\
\text { débutant }\end{array}$ & 64.3 & 18.7 & 9.3 & 7.5 & & \\
\hline
\end{tabular}

Le cas Harpe 1A contraste de manière assez claire avec les deux autres : bien que dans LO une partie initiale de la leçon soit exclusivement consacrée à des MT, pour les autres leçons les occurrences MI sont largement distribuées sur l'ensemble de la leçon, et les moments où ces savoirs MI ne sont pas couplés avec d'autres savoirs sont assez nombreux.

Les moyennes concernant les deux autres catégories de savoirs sur la lecture de partition (ML) et sur la préparation du travail à la maison $(\mathrm{MH})$ sont peu élevées pour l'ensemble des cas (autour du 10\% des occurrences), et le lien avec l'une ou l'autre des conceptions ne se présente pas de manière aussi tranchée. Le cas Violoncelle 1A se distingue de manière claire des deux autres, aux chiffres très semblables (lorsque l'on ne considère que les leçons auprès des débutants pour Violoncelle 1B). Comme nous le verrons dans les analyses qualitatives, le comportement de ces deux catégories de savoirs s'explique davantage par rapport à l'expérience d'enseignement, les deux derniers cas (Harpe 1A et Violoncelle 1B) correspondant à des enseignants en formation en fin d'études de $2^{\mathrm{e}}$ année du master.

Ces données nous offrent donc un premier aperçu tant de l'organisation que de la distribution générale des quatre catégories de savoirs (MT, MI, ML, $\mathrm{MH}$ ). Leur articulation avec des données qualitatives permet de mieux comprendre les choix didactiques de ces enseignants en formation et de répondre plus finement à nos deux questions de recherche. 


\section{Premier cas : Violoncelle 1 A (STE)}

Le cas de Ste (enseignante Violoncelle 1A) est celui qui semble s'inscrire de manière plus claire dans une orientation " technique PUIS interprétation ». Un indice de l'orientation technique de son enseignement en début du semestre est le fait que dans LO, un nombre très important d'occurrences MT ne sont pas engagées avec d'autres contenus ( $c f$. figure 1, histogramme 1). Cette configuration se répète pour L2 et L4, et de manière plus nuancée en L3 (figure 2). Chez Ste, une bonne moitié de leçon est consacrée à des exercices sur la gamme dans le but de travailler : la position de la main gauche, la posture générale de tenue de l'instrument, la tenue de l'archet à la main droite et la justesse du son produit. Les occurrences MI au début de L3 sont liées à ce dernier aspect, puisque Ste tente d'articuler les aspects techniques de justesse du son à des aspects esthétiques d'un « beau son » et du " ressentir» de la sonorité de l'instrument. La figure 2 montre l'histogramme de la distribution par minute des savoirs définis par l'enseignante en formation, de L1 à L4. Puis, dans les extraits 1 et 2, l'analyse permet de montrer comment se joue cette orientation « technique PUIS interprétation » par rapport aux deux topoï musicien-instrument et respectivement musicien-œuvre.

\section{Une construction de l'interprétation orientée par la maîtrise technique}

Ste oriente son élève, qui est un débutant adulte, vers un travail postural qui concerne la position des mains sur la touche et sur la tenue et le contrôle de la quantité d'archet, mais qui vise également la prise de conscience du rapport musicien-instrument. Les apports techniques ne sont pas seulement liés à une dimension posturale et gestuelle dans leurs aspects formels et externes. Ils le sont également au travail sur la dimension « sensible » du corps et au fait que le musicien doit être en mesure de réguler le jeu à partir des retours sonores de ses actions. L'autorégulation concerne la conscience proprioceptive de la tenue de l'instrument liée au postural, mais aussi des aspects qui paraissent peu évidents aux débutants comme celui du rôle que joue la respiration dans la dynamique des gestes ( $c f$. extrait 1, 22'57):

Extrait 1, L0 : Travail sur le « corps sensible»

\begin{tabular}{|l|l|}
\hline $22 ' 57$ & $\begin{array}{l}\text { Ste : Parce que tu pars de rien (montre un } \\
\text { contre-exemple de jeu sans préparer la respi- } \\
\text { ration); c'est un peu dur, pour tout le monde } \\
\text { c'est dur, }\end{array}$ \\
\hline $23^{\prime} 00$ & $\begin{array}{l}\text { Ste : ... avec une respiration, ça aide (Ste prend } \\
\text { une grande respiration et ils jouent en duo) }\end{array}$ \\
\hline
\end{tabular}

Figure 2 : Violoncelle 1A - Histogrammes de la distribution par minute des savoirs définis par l'enseignante en formation (Ste), leçons $\mathrm{Ll}$ à L4
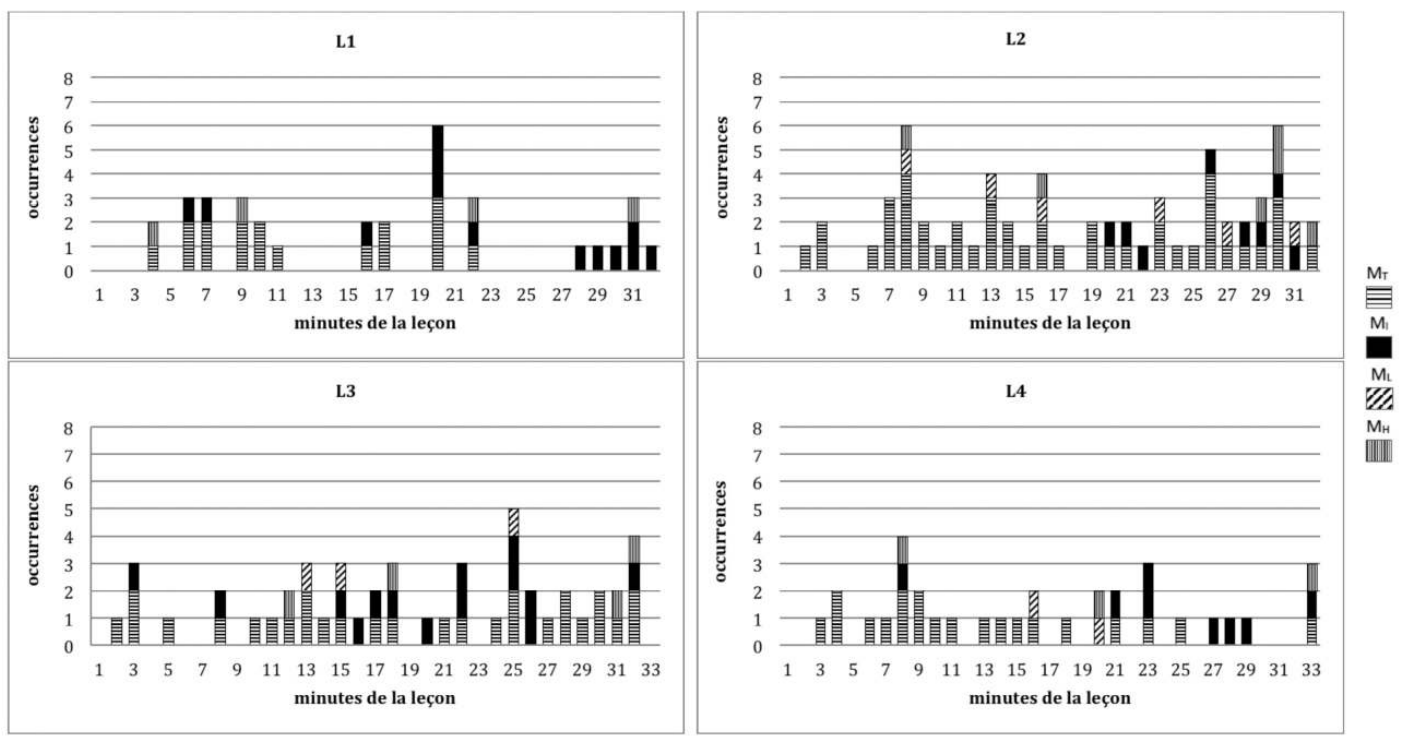
C'est dans ce contexte qu'émergent certaines des occurrences MI liées au rapport entre un geste efficace et la production d'un bon « son rempli » (cf. extrait 2, 16'00) qui pourra par la suite s'articuler avec les dimensions esthétiques d'un « beau son »:

Extrait 2, L0 : Dimensions technique et esthétique de la production sonore

\begin{tabular}{|l|l|}
\hline $16 ' 00$ & $\begin{array}{l}\text { Ste : Voilà... et la dernière chose en fait quand } \\
\text { t'arrives à... (geste) pense à remplir ton son } \\
\text { toujours plus c'est ton oreille qui doit te dire } \\
\text { quel geste tu dois avoir mais c'est bien quand tu } \\
\text { arrives à sentir que ton coude (elle joue) il est } \\
\text { toujours en mouvement }\end{array}$ \\
\hline $19^{\prime} 37$ & $\begin{array}{l}\text { Ste : On va essayer d'avoir un legato quelque } \\
\text { chose d'un peu plus uniforme pour après, rajou- } \\
\text { ter les attaques que tu veux... }\end{array}$ \\
\hline
\end{tabular}

De même, le travail technique sur le legato (cf. extrait 2, 19'37) est celui qui autorisera la dimension interprétative ( rajouter les attaques que tu veux »). Dans les leçons de ce cas, l'effet interprétatif découle presque systématiquement de la maîtrise d'un élément technique pour les occurrences MI. Cela avec une autre conséquence remarquable, qui est l'attribution de la responsabilité interprétative au topos musicien-instrument, comme le montre l'enchaînement de l'extrait 3 :

Extrait 3, L1 : La technique d'archet comme préalable à l'interprétation de l'étude

\begin{tabular}{|l|l|}
\hline $10^{\prime} 42$ & $\begin{array}{l}\text { Ste : On réessaie de mobiliser ton avant-bras } \\
\text { dans l'espace comme on avait fait la dernière } \\
\text { fois, un peu des ronds. Essaie d'avoir un contact } \\
\text { avec... (Geste) Tu peux t'entraîner à faire plus } \\
\text { large... Arrondis ton pouce et là tu allonges } \\
\text { Ouvre bien la paume de ta main. On y va? }\end{array}$ \\
\hline $13^{\prime} 10$ & $\begin{array}{l}\text { Ste : Attention ta main gauche, tu resserres beau- } \\
\text { coup trop les doigts (Ste replace la main gauche } \\
\text { de l'élève) }\end{array}$ \\
\hline $15^{\prime} 40$ & $\begin{array}{l}\text { Ste : Amuse-toi, profite de ce SOL à vide qui } \\
\text { résonne (corde à vide) }\end{array}$ \\
\hline
\end{tabular}

Dans cet extrait, une série de régulations techniques de type postural débouchent sur l'injonction de s'amuser avec les effets sonores de l'instrument. En ce sens, la dimension "interprétation » se joue chez cette enseignante, notamment, dans la relation du jeu musical et d'un corps sensible aux effets du jeu de l'instrument.

\section{L'interprétation de l'œuvre : une responsabilité laissée à l'élève}

En général, cette enseignante en formation (Ste) cherche à relier, de manière cohérente, les objets d'enseignement abordés dès la phase d'échauffement à ceux qu'elle souhaite travailler durant la leçon. S'appuyant fortement sur la méthode Feuillard (1970), les exercices et études permettent à Ste d'introduire des objets habituels de technique instrumentale. Caractéristiques de ces méthodes, les petites études qui émaillent les divers exercices permettent aux élèves d'être en contact également avec des éléments relevant de l'œuvre musicale, tout en exerçant ces savoirs techniques. Ces ressources didactiques font écho à la structure canonique des leçons qui organise l'activité de l'élève dans une suite échauffement-exercices (ou études) - jeu d'une ouvre, que Ste suit de très près en L0, L2, L3 et L4 (cf. figure 2).

Lorsque l'enseignante en formation traite des contenus MI liés au jeu d'une œuvre, avec des éléments tels que le phrasé (L1), les nuances (L1; L2; L3) ou le rythme (L2, L4), ces éléments sont en lien avec l'interprétation de ces petites pièces musicales choisies pour l'élève, qu'elle aborde dans le dernier tiers de leçon. Dans L1, par exemple, la dimension proprement interprétative ne sera explicitement travaillée que dans le dernier tiers de la leçon, à partir de 22'45, lorsque la pièce Melancolia de Chopin ${ }^{12}$ est abordée. Un des indices de cette bascule vers l'interprétation d'une pièce en tant qu'œuvre est le fait que, dès le début, Ste joue avec son élève. Durant ces moments des leçons, le jeu conjoint constitue un indice du changement de milieu.

La dimension interprétative se construit alors dans une alternance entre des aspects structurels appartenant à l'œuvre et des choix d'interprétation que l'élève devrait faire ressortir. La demande de Ste en $27^{\prime} 05$ ( $c f$. extrait 4 ) est en ce sens exemplaire de son approche : d'une part, elle pointe la phrase musicale comme élément qui structure l'œuvre performée et, d'autre part, à travers sa demande de définir les phrases, elle signifie à l'élève que cette action relève de la responsabilité de l'interprète dans le but de donner au jeu plus de relief ( $c f$. son geste de contreexemple indiquant l'uniformité du jeu comme un problème à résoudre), cela sur la base de choix tels que les contrastes de nuances (28'00: « tu me le joues vraiment fort $»$ ) : 
Extrait 4, L1 : Interprétation de l'œuvre

\begin{tabular}{|l|l|}
\hline $27 ’ 05$ & $\begin{array}{l}\text { Ste : Est-ce qu'on pourrait définir les phrases } \\
\text { musicales? Parce que là c'est tout (fait un geste } \\
\text { qui semble signifier que tout est « plat ») et on } \\
\text { peut ainsi structurer les choses, et après tu peux } \\
\text { proposer. }\end{array}$ \\
\hline $27^{\prime} 35$ & $\begin{array}{l}\text { Ste : La première phrase, elle irait jusqu'où? } \\
\text { (élève signale un endroit de la partition, puis } \\
\text { joue le début de la deuxième phrase) } \\
\text { Ste : Oui (Ste chante les deux premières mesures } \\
\text { la mélodie, puis joue l'accompagnement) }\end{array}$ \\
\hline $28^{\prime} 00$ & Ste : On essaie ça, tu me le joues vraiment fort? \\
\hline
\end{tabular}

L'analyse du déroulement général des leçons ( $c f$. figure 2) montre cependant que ces éléments interprétatifs finissent le plus souvent par être suspendus au profit, soit d'enjeux locaux liés à la résolution de problèmes de technique instrumentale, soit attribués par Ste à la sensibilité musicale de l'élève. En filigrane, l'on repère chez Ste un leitmotiv qui apparaîtra plusieurs fois durant les leçons : celui du plaisir associé à certaines caractéristiques du jeu

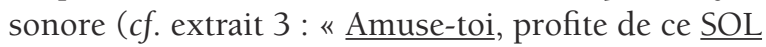
à vide qui résonne; ca sonne super bien donc tu fais sonner $»$ ).

Une approche similaire peut être constatée en L3 à propos du jeu d'un menuet : ci-après, dans l'extrait 5, la gestion des longueurs d'archet par rapport à la durée des notes écrites (20'05) est posée comme préalable à l'usage efficient de la pulsation choisie pour la pièce (21'23), qui par ailleurs doit être corporellement anticipée et ressentie (19'11), cela avec une fonction très proche de celle que nous avons soulignée pour la respiration comme moteur du coup d'archet (cf. extrait 1 ).

La construction de l'interprétation orientée par les caractéristiques de l'œuvre est plutôt un phénomène rare dans les leçons de cette enseignante en formation. Dès lors, il n'est pas étonnant de voir émerger la question de l'interprétation surtout dans L4, leçon qui marque une fin de cycle à la fois dans la dimension purement temporelle (fin de l'année scolaire), en rapport avec la logique subjacente à la conception « technique PUIS interprétation » qui est à son tour renforcée par la structure ternaire qui organise les formes scolaires d'apprentissages. Cette leçon ayant eu lieu à la fin du mois de mai, le parcours de Ste avec son élève arrive à une phase où l'enjeu semble être celui de mobiliser l'ensemble des acquis techniques au profit d'un jeu instrumental qui autorise le plaisir et l'expressivité. Le travail qu'elle réalise pour accompagner son élève, dans le décodage puis dans la réorganisation interprétative de l'œuvre, montre qu'elle dispose d'un certain nombre d'outils conceptuels et didactiques pour construire la posture d'interprète, mais qu'elle n'arrive pas encore à en tirer parti : elle en diminue souvent la portée au profit d'une attente forte liée à l'émergence chez l'élève d'une sensibilité esthétique générale qui ne tient pas forcément compte des œuvres jouées.

\section{Extrait 5, L3 : Pulsation, rythme} et interprétation du menuet

\begin{tabular}{|l|l|}
\hline $19 ' 11$ & $\begin{array}{l}\text { Ste : Ok, on s'arrête là? ... Juste dernière chose : } \\
\text { avant de partir, tu peux faire trois temps (Ste } \\
\text { se balance) 2, 3 (elle joue avec l'élève), voilà et } \\
\text { après c'est bon }\end{array}$ \\
\hline 20 '05 & $\begin{array}{l}\text { Ste : Et là c'est toujours le risque d'écourter (Ste } \\
\text { pointe l'archet) la dernière noire. Tu fais (Elle } \\
\text { joue) ... parce que tout simplement tu ne sépares } \\
\text { pas bien ton archet (geste) en combien? } \\
\text { (l'élève répond « trois ») donc il faut que tu } \\
\text { sépares en? trois. En fait, ce travail aussi peut } \\
\text { t'aider. Comme ça il te reste vraiment un tiers } \\
\text { d'archet (geste). Pas moins. Il ne faut pas moins, } \\
\text { surtout pour la fin de l'archet pour faire le } \\
\text { changement. Si tu as moins d'un tiers d'archet, tu } \\
\text { risques d'écourter la noire. }\end{array}$ \\
\hline 21 '23 & $\begin{array}{l}\text { Ste : Maintenant que tu as bien la pulsation et } \\
\text { tout ça tu peux peut-être oser plus de nuances. }\end{array}$ \\
\hline
\end{tabular}

\section{HARPE 1A (STI) : L'AUVRE COMME SOURCE DES APPORTS TECHNIQUES}

\section{Une construction de l'interprétation orientée par l'œuvre}

L'analyse de l'ensemble des leçons (cf. figure 3) données par cette enseignante (Sti) permet de constater que son projet d'enseignement s'organise à partir d'une autre conception : « interprétation ET techniques afférentes ", et que ses leçons suivent en conséquence une version particulière de la structure canonique " échauffement-exercices-interprétation ». Cette enseignante aborde certes de très nombreux apprentissages techniques ( $40 \%$ de MT en moyenne sur l'ensemble de ses leçons), mais ces derniers sont toujours dans un rapport étroit avec les dimensions interprétatives (42.2\% de MI en moyenne sur l'ensemble des leçons), cela dans un équilibre qui suggère davantage d'articulations. La distribution des rapports 
MT/MI semble être permanente pour les leçons de cette enseignante, qui introduit les éléments liés à l'interprétation musicale de l'œuvre très tôt dans les leçons (L1 min. 4, L2 min. 3, L3 min. 2 et L4 min. 5).

Les données analysées mettent en évidence deux grands ensembles dans le rapport MT/MI. Un premier ensemble, proportionnellement le plus important des deux en nombre d'occurrences, concerne les savoirs MI comme construction d'un rapport interprétatif aux œuvres; un second ensemble concerne les savoirs MI développés à partir de tâches d'improvisation musicale, visant la construction d'un rapport de l'élève à son instrument en termes d'expressivité du jeu musical. L'importance du premier ensemble signe une particularité remarquable quant à l'enseignement dans ces leçons. Contrairement aux stratégies de travail de la conception " technique PUIS interprétation ", les savoirs MT ne semblent pas constituer des préalables au travail interprétatif. Cette particularité tient justement à une stratégie de travail basée sur l'interprétation d'œuvres, choisies par l'enseignante en fonction de certains défis techniques qu'elle souhaite faire travailler à son élève. Par exemple, dans le cas de LO, le défi consiste à effectuer des suites d'arpèges en évitant des pauses ou des interruptions liées aux déplacements des doigtés ou à l'enchaînement main gauchemain droite. C'est ce projet d'articulation de savoirs MI et MT qui peut être constaté à partir des expressions indiciaires « transition", " point culminant [de la phrase] » ou « tension de la phrase » (cf. extrait 6$)$ :
Extrait 6, Harpe-L0 : Retour sur des dimensions interprétatives à partir du travail technique

\begin{tabular}{|l|l|}
\hline $18 ' 19$ & $\begin{array}{l}\text { Sti : A droite, comment tu places, déjà? (Pointe } \\
\text { la partition). Là comment tu fais ta transition? }\end{array}$ \\
\hline $18^{\prime} 48$ & $\begin{array}{l}\text { Sti : Après, dans notre phrase, on a une évo- } \\
\text { lution du tempo. On va jusqu'où dans cette } \\
\text { phrase? Quel est le point culminant? } \\
\text { El : Larpège }\end{array}$ \\
\hline $19^{\prime} 04$ & $\begin{array}{l}\text { Sti : Le début est là (pointe la partition) et il faut } \\
\text { tenir la tension de la phrase. }\end{array}$ \\
\hline $19^{\prime} 15$ & Sti : (chante un exemple en crescendo) \\
\hline
\end{tabular}

L'analyse des leçons suivantes confirme que cette modalité d'articulation MT/MI constitue un projet chez Sti, dans lequel l'interprétation musicale passe par l'apprentissage d'un premier processus consistant à « décoder et comprendre le fonctionnement structurel de l'œuvre ». Dans ce projet, il ne s'agit pas tant de la dimension technique liée au solfège, que d'aspects impliquant un premier niveau de «textualité de l'écriture musicale », en particulier, l'identification des phrases musicales dans l'œuvre:

Extrait 7, Harpe-L1 : Identification des structures constitutives de l'œuvre : les phrases musicales

\begin{tabular}{|l|l|}
\hline $05^{\prime} 48$ & $\begin{array}{l}\text { Sti : Donc il y a une première fin qui se trouve } \\
\text { ici (pointe et souligne la partition). Tu es à cheval } \\
\text { sur la fin de cette grande phrase-là et le début de } \\
\text { la suivante. (pointe la partition). }\end{array}$
\end{tabular}

Figure 3 : Harpe 1A - Histogrammes de la distribution par minute des savoirs abordés par l'enseignante en formation (Sti), leçons L1 à L4.
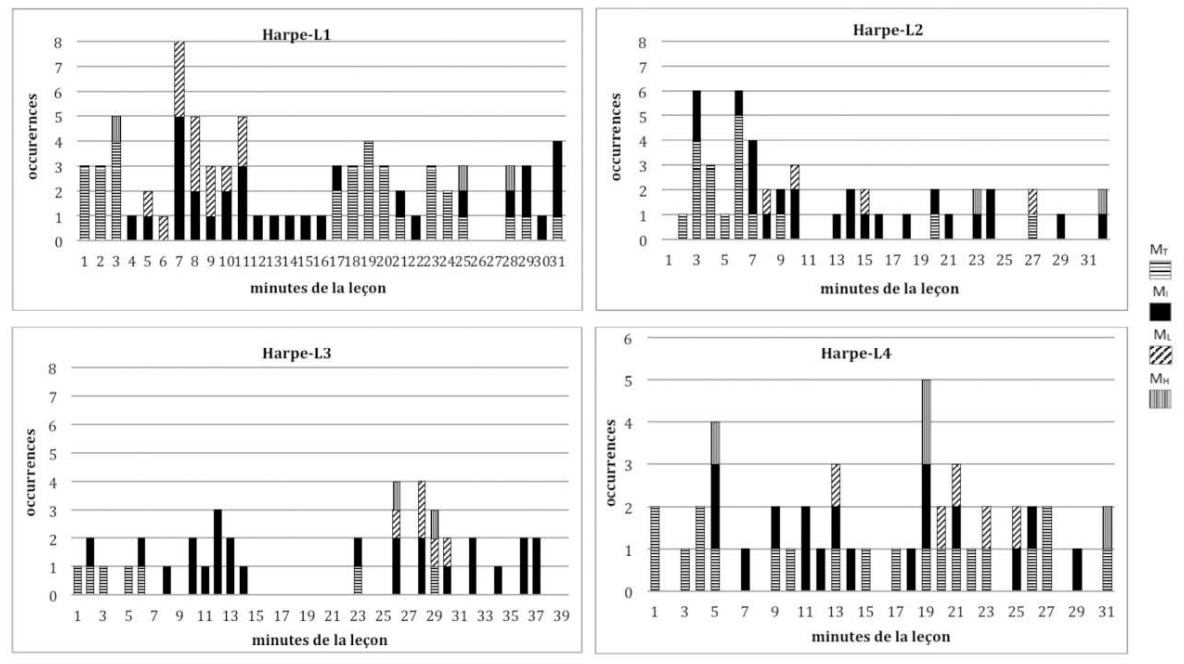
Cela marquant une orientation interprétative de ces identifications, qui de plus, est à la charge de l'élève, sous forme de dévolution. Ces constantes orientations de l'enseignante visent, en effet, à terme la construction d'un phrasé musical, et l'interprétation de l'œuvre peut se structurer au fil des leçons :

Extrait 8, Harpe-L3 : Construction du phrasé dans l'interprétation de l'œuvre

\begin{tabular}{l|l}
10 & \\
& Sti : [De quoi on a déjà parlé, au niveau de ce \\
& qui est musical et interprétation? \\
& Pi : Des phrasés : faire une grande phrase sur \\
la première partie et faire des respirations, là \\
(montre sur la partition)] \\
Sti : On avait parlé de qu'est-ce que ça veut dire, \\
faire une phrase, est-ce que tu te souviens? \\
Pi : Dans le ressenti [...] Avec une intention de \\
finir la phrase. \\
Sti : C'est juste, et pour donner l'impression que \\
le son tienne et que la phrase tienne et continue, \\
il faut penser qu'il y a du lien entre les notes.
\end{tabular}

Le projet enseignant ne laisse pas pour autant de côté le topos de la sensibilité du musicien. Dans l'extrait 8 ci-dessus, les expressions « ressenti » et « intention » utilisées par l'élève indiquent qu'il y a lieu à s'orienter vers la construction de ce topos.

\section{La construction d'un rapport expressif à l'instrument}

Ce second topos est également très présent dans la plupart des leçons et fait même l'objet d'une exception autorisée par la formatrice dans la leçon L3 durant laquelle Sti, qui n'a pas réussi à gérer le temps de la leçon durant le travail sur le technique, obtient de sa formatrice de lui accorder presque « 10 minutes de plus pour le travail sur l'improvisation ». Il est construit à partir d'une stratégie enseignante qui alterne deux objets d'enseignement distincts. D'une part, l'enseignante insiste sur la production d'une sonorité autant au niveau des qualités de chaque son, que des enchaînements de sons :

Extrait 9 Harpe-L0 : Construction de la sonorité, enchaînement des sons et qualités du son

01'18 Sti : Aujourd'hui, on va centrer notre attention sur avoir le même son (unité), dans chacun des doigts.
05’01 Sti : J'aimerais qu'on améliore: dans les aigus, même (intensité) sonore que pour les médiums; ça a tendance à faire (joue exemple) ... un peu «pâle »

D'autre part, vers la fin de la leçon, dans la troisième partie de la structure tripartite déjà mentionnée, l'enseignante vise la construction de gestes de jeu de l'instrument, notamment à partir de l'improvisation comme moyen de "décoller » l'élève des contraintes de l'œuvre et celles de la partition :

Extrait 10, Harpe-L2 : Construction de la sonorité, enchaînement des sons et qualités du son

\begin{tabular}{|l|l|}
\hline $28 ' 51$ & $\begin{array}{l}\text { Sti : (Aujourd'hui, sur notre gamme pentato- } \\
\text { nique) on va chercher à mettre le bon geste. On } \\
\text { va chercher à faire une musique saccadée qui } \\
\text { nous demande un geste saccadé. }\end{array}$ \\
\hline 29 '45 & $\begin{array}{l}\text { Sti : Donne-moi également (un signe ou un geste } \\
\text { qui concrétise) la fin (de l'impro). }\end{array}$ \\
\hline 30 '50 & $\begin{array}{l}\text { Sti : Dans ce jeu-là, on ne cherche pas un rendu } \\
\text { sonore; on cherche un moyen. On cherche le } \\
\text { chemin, pas l'arrivée. }\end{array}$ \\
\hline 31 '03 & $\begin{array}{l}\text { Sti : On ne cherche pas (à réaliser) une } \\
\text { mélodie, mais une façon de la faire. Si je veux } \\
\text { une musique saccadée, je cherche le geste qui } \\
\text { correspond. }\end{array}$ \\
\hline 31 '28 & \begin{tabular}{l} 
Sti : Exagérer un geste. \\
\hline
\end{tabular} \\
\hline
\end{tabular}

À noter que l'enseignante joue également sur des variables didactiques liées aux contenus travaillés précédemment par rapport à l'œuvre. Dans L3, à propos du rapport tonique - dominante, elle pose comme contrainte des phrases improvisées en insistant pour que l'élève termine l'improvisation sur la tonique :

Extrait 11, Harpe-L3 : Improvisation et caractère

05'15 Sti : Aujourd'hui, on va faire une impro, mais pas en pentatonique; plutôt en tonal et rester en Do Majeur, parce que c'est plus facile. On va jouer toutes les deux : tu vas jouer une mélodie et je vais faire l'accompagnement. C'est toi qui vas décider quand on commence et s'arrête et quand on s'arrête, fais en sorte que ce soit sur un Do. Pour le caractère de ta mélodie, on a le choix entre la joie, la tristesse, la colère, la peur et la tendresse.

Elle sollicite ainsi les représentations de son élève sur le caractère musical, mais en lui fournissant 
également des pistes concernant «l'écriture musicale» tonale. Contrastant avec le premier cas abordé (Violoncelle-IA), ici le topos musicien-instrument est développé dans un lien plus étroit avec l'écriture musicale.

Chez cette jeune enseignante, la stratégie d'enseignement basée sur la conception « interprétation de l'œuvre ET techniques afférentes » conduit vers une gestion assez différente de l'articulation technique/interprétation, les savoirs concernant cette dernière étant souvent la référence principalement co-construite durant les leçons. L'orientation sur l'interprétation de l'œuvre, qui constitue la principale visée de ses enseignements, n'empêche pas pour autant Sti d'aborder le topos musicien-instrument, à partir de tâches d'improvisation dont l'objectif est davantage le développement d'un geste expressif qu'un rendu sonore en termes de maîtrise de la technique instrumentale.

\section{VIOLONCELLE $1 \mathrm{~B}$ (STH)}

\section{L'interprétation comme rapport personnel au jeu instrumental}

Le cas Violoncelle 1B, s'inscrit également dans la conception « technique PUIS interprétation » comme le premier cas, mais nous permet aussi d'aborder la question des articulations MT/MI à partir de la variable élève débutant versus élève avancé. En effet, les leçons observées de l'enseignant en formation Sth ne concernent pas toujours le même élève. L0, L2 et L4 portent sur un même élève, un débutant de 9 ans. L1 concerne un adulte de niveau débutant et L3 un adulte de niveau avancé. La durée des leçons varie entre 28 et 29 minutes, sauf pour L3 qui en dure 16. Ces discontinuités concernant l'âge et le niveau des élèves auprès desquels Sth s'exerce comme enseignant, pourraient être considérées comme une limite méthodologique de l'étude. Nous considérons, au contraire, qu'elles nous permettent d'approcher les profils d'enseignement de Sth par rapport à un élément clé des processus de professionnalisation : la capacité de l'enseignant à adapter son agir aux caractéristiques spécifiques des contextes d'activité.

Comme pour les autres cas de cette étude, dans celui-ci les occurrences entre MT et MI sont dans un rapport contrasté favorisant MT, sauf pour L3 où le rapport est totalement inversé : (cf. tableau 3). Dans les 4 leçons avec un élève débutant, les rapports MTMI sont sensiblement proches de ceux étudiés pour Violoncelle $1 \mathrm{~A}$, avec des questions relatives à la tenue de l'instrument, à la posture générale, au maniement de l'archet, etc. Cependant, chez Sth des occurrences MI émergent plus tôt dans la leçon et sont plus souvent articulées avec les MT et les deux autres catégories de savoirs. Si l'on considère uniquement les occurrences relatives aux leçons avec les élèves débutants, les pourcentages sont proches de ceux du premier cas. Comme nous l'avons souligné, l'inversion des pourcentages dans L3 permet de montrer que les conceptions des rapports MT/MI ne sont pas nécessairement un facteur rigide présidant à toute décision didactique d'un enseignant; il s'agit davantage de « schèmes de lecture » que l'enseignant mobilise en fonction des caractéristiques des contextes : dans le cas de L3, le fait qu'il s'adresse à une élève avancée modifie en profondeur les enjeux d'enseignement de la leçon.

Un autre élément caractéristique de l'enseignement de Sth est la proportion très importante de moments de la leçon durant lesquels il n'y a pas de gestes de définition de sa part (cf. figure 4), ce qui contraste avec les profils des deux autres cas. Ces moments traduisent un rapport beaucoup plus détendu à ses élèves, qui disposent avec cet enseignant de plus de place pour assumer et développer leurs responsabilités des tâches.

\section{La qualité sonore comme condition d'un rapport au jeu instrumental}

À l'exception de L3, pour l'ensemble des leçons menées auprès d'élèves débutants les rapports MT/MI semblent s'organiser selon cette même tendance mise en place en LO : dans les leçons les aspects interprétatifs orientés par le technique concernent, notamment, une attention aux contours de la mélodie, aux attaques des notes, aux liaisons et aux nuances. L'enseignant en formation montre ainsi que l'interprétation reste à la fois dépendante du maniement correct de l'instrument mais qu'elle peut être orientée vers des résultats d'interprétation de la pièce, qui permettent à l'élève de comprendre pourquoi il doit prioritairement les travailler. Dans la figure 4, l'on constate qu'en Ll les occurrences MI sont très peu nombreuses et fortement dépendantes de l'orientation technique de la leçon. Cependant, Sth articule 


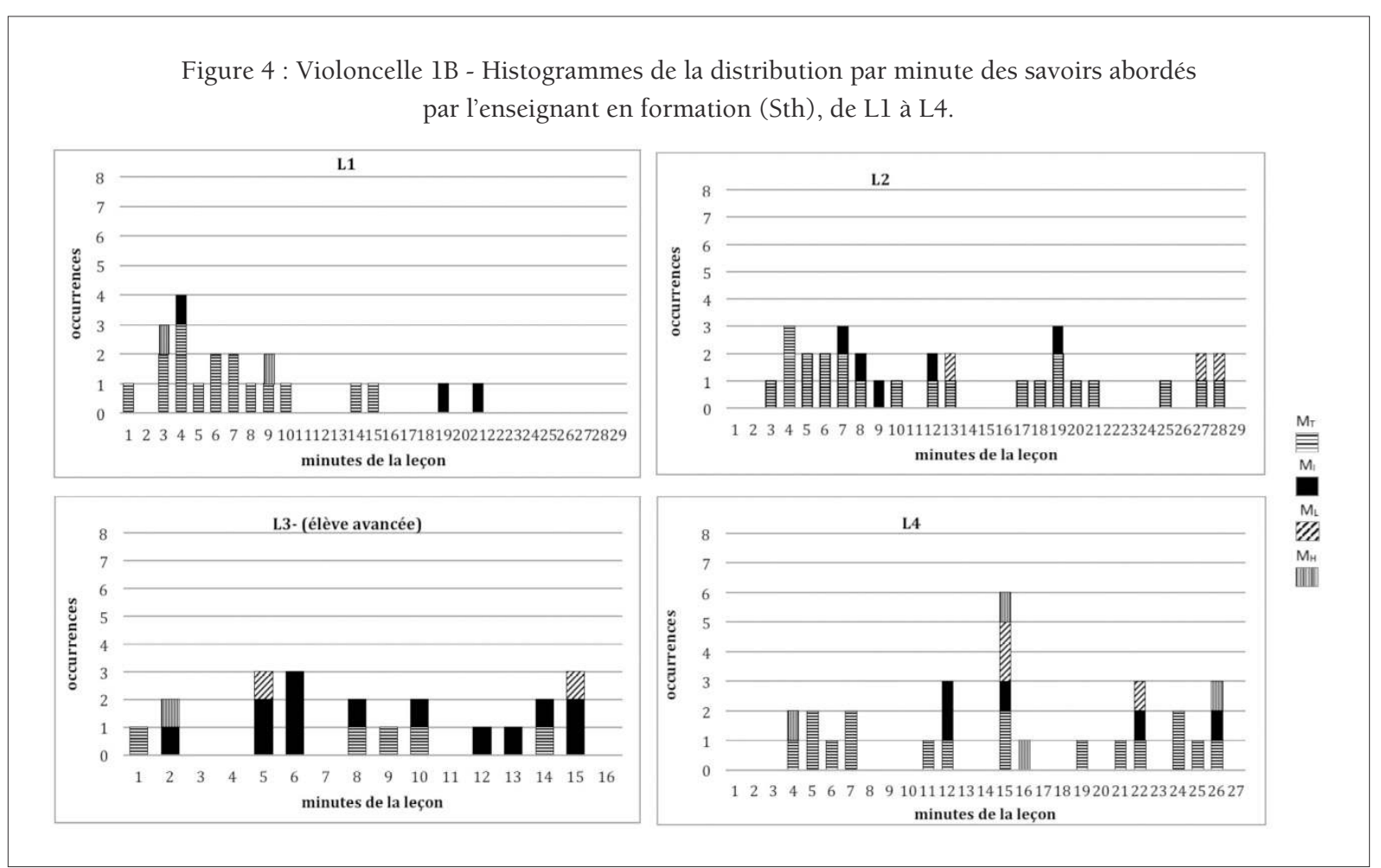

très souvent les exigences techniques à la dimension esthétique en termes de sensibilité (3'00) et de qualité sonore (3'10) :

Extrait 12, Violoncelle-L1 : Construction de la sonorité et qualités du son

\begin{tabular}{|l|l|}
\hline 3'00 & $\begin{array}{l}\text { Sth : Ça, on va le travailler chaque leçon pour } \\
\text { trouver les sensations. }\end{array}$ \\
\hline 3'10 & $\begin{array}{l}\text { Sth : En fait il faut être sur } 2 \text { archets, toujours } \\
\text { tirer pousser, en fait, sur 4 temps. (Sth lui pro- } \\
\text { pose un exercice pour la gestion des longueurs } \\
\text { d'archet, en lui demandant d'appuyer vraiment } \\
\text { fort sur la corde à vide). } \\
\text { Sth : Appuie vraiment, sur le sol... ça va pas être } \\
\text { joli, [plutôt] vraiment dégueulasse. }\end{array}$ \\
\hline
\end{tabular}

Cet effort d'articulation est également visible pour l'ensemble des leçons, dont les minutes «sans action de définition enseignante » constituent un indice fort de la place assez importante qu'il laisse au jeu de l'élève pour laisser émerger des « choix interprétatifs» de l'instrumentiste. Certes, comme le montre l'extrait 13, du côté enseignant les choix de tempo font partie d'une stratégie liée aux possibilités « techniques » de jouer le morceau : si le tempo est trop rapide, les capacités techniques actuelles de l'élève l'empêcheront de le jouer. Mais, en même temps, du côté de l'élève, sont travaillés des aspects qui relèvent aussi du fait que l'instrumentiste doit faire souvent des choix (8'20) et adapter et intérioriser certains aspects des morceaux joués (9'01):

Extrait 13, Violoncelle-L2 : Dévolution du jeu interprétatif et étayage enseignant

\begin{tabular}{|l|l|}
\hline $6 ' 57$ & $\begin{array}{l}\text { Sth : Chante la mélodie (l'élève chante avec un } \\
\text { tempo modéré). }\end{array}$ \\
\hline 7'08 & $\begin{array}{l}\text { Sth : Est-ce le bon tempo? (l'élève chante avec } \\
\text { un tempo très rapide). }\end{array}$ \\
\hline $8 ' 20$ & $\begin{array}{l}\text { Sth : Moi, j'ai préféré la première version, pas } \\
\text { trop vite. }\end{array}$ \\
\hline 9'01 & Sth : Imagine le tempo dans ta tête. \\
\hline
\end{tabular}

Ces choix techniques trouvent leur raison d'être dès la minute 21 ( $c f$. extrait 14), à partir de laquelle l'enseignant en formation et l'élève jouent ensemble le morceau (min. 21 à 24). La leçon L4 confirme la conception de cet enseignant en formation par deux aspects, le premier étant la répartition constante des MT sur l'ensemble de la leçon qui souligne l'importance du technique dans son enseignement auprès des débutants. Alors que le second est la distribution 
stratégique des 4 occurrences MI qui marquent les visées interprétatives de ces acquis techniques:

Extrait 14, L4 : Articulation finale des acquis techniques en fonction de visées interprétatives

\begin{tabular}{|l|l|}
\hline 4'34 & $\begin{array}{l}\text { Sth : Pas jouer comme un tornado mais comme } \\
\text { une brise }\end{array}$ \\
\hline 11 '03 & $\begin{array}{l}\text { Sth : On rejoue depuis le début avec une } \\
\text { sonorité plus large et plus legato (il joue pour } \\
\text { montrer) }\end{array}$ \\
\hline $12^{\prime} 43$ & Sth : Plus fort ou piano en jouant la pièce \\
\hline 14 '30 & $\begin{array}{l}\text { Sth : Pour la prochaine fois, tu me fais l'exer- } \\
\text { cice avec les mêmes notes liées et plus fort. Tu } \\
\text { peux choisir tes dynamiques. }\end{array}$ \\
\hline 21 '00 & $\begin{array}{l}\text { Sth : Là il y a des points sur les notes ... je te } \\
\text { montre deux versions, laquelle tu préfères? }\end{array}$ \\
\hline 23 '09 & $\begin{array}{l}\text { Sth : L'élève : « comme un bateau sur la mer } \\
\text { quand il y a des grosses vagues » (se référant } \\
\text { au maniement de l'archet qui donne un certain } \\
\text { résultat sonore) }\end{array}$ \\
\hline
\end{tabular}

Les interventions de l'enseignant concernant les occurrences MI dans L4 (cf. extrait 14) montrent, en effet, les différents aspects des savoirs interprétatifs et leurs articulations avec l'objectif d'une maîtrise technique de l'instrument. Mais, contrairement au cas Harpe 1A, l'œuvre est davantage un prétexte pour travailler des aspects relevant, notamment, du topos « rapport sensible au jeu instrumental».

Le statut de "débutant » des élèves dans les leçons L0, L1, L2 et L4 peut constituer une explication de la prépondérance observée de la conception « technique PUIS interprétation » guidant les choix didactiques de cet enseignant tout comme ceux de l'enseignante du premier cas étudié. L'apprentissage du jeu avec des instruments non tempérés, comme le violoncelle, marque ainsi une nette différence avec la harpe qui se manifeste, notamment, par une approche plus " tendue » aux savoirs de type technique. C'est ce même aspect qui explique l'inversion des rapports MT/MI que présente la leçon L3 de cet enseignant en formation, car menée auprès d'une élève avancée.

Cette leçon L3 constitue en fait un exemple de continuité de la conception de Sth, par rapport à laquelle à partir d'un certain niveau de maîtrise technique de l'instrument, l'enseignant donne la possibilité à l'élève de développer ses propres interprétations des œuvres. La durée de la leçon est un indice intéressant : non seulement les élèves avancés ont plus de moyens pour comprendre rapidement les attentes de l'enseignant en formation mais, de plus, la définition du travail à faire en dehors de la leçon se trouve également être plus rapide et efficace. De ce fait, le travail de l'enseignant consiste, essentiellement, à définir la nature et l'empan du travail interprétatif que l'élève prendra en charge en dehors du temps de leçon. Dans L3, les occurrences MI largement majoritaires se répartissent sur toute la durée de la leçon, chaque regroupement étant suivi d'ailleurs par une plage de dévolution importante. Comme on peut le constater dans l'extrait 24 , du point de vue topogénétique, l'enseignant et son élève se trouvent dans des rapports horizontaux plus proches de l'échange entre experts que de celui entre l'expert et le novice. L'articulation avec les aspects techniques s'inverse dans cette leçon, ceux-là étant dépendants des besoins interprétatifs. Comme le montre l'extrait suivant, l'enseignant en formation aborde des aspects relevant de l'œuvre mais la majorité des occurrences MI de cette leçon s'articule davantage avec une approche de l'interprétation en termes de choix subjectifs :

Extrait 15 : Articulation technique-interprétation comme choix personnel

\begin{tabular}{|l|l|}
\hline $7 ' 35$ & $\begin{array}{l}\text { Sth : Est-ce ce que cela te dirait de jouer un } \\
\text { peu plus vers la touche? }\end{array}$ \\
\hline 9'10 & $\begin{array}{l}\text { Sth : Est-ce que tu reprends? Moi je trouve- } \\
\text { rais plus joli mais c'est un choix personnel (il } \\
\text { montre les coups d'archet) et puis tu reprends } \\
\text { l'élan... }\end{array}$ \\
\hline $11^{\prime} 00$ & $\begin{array}{l}\text { Sth : ... je te donne deux possibilités : soit } \\
\text { moins fort, plus intime ou bien tu confirmes } \\
\text { ce qui est déjà dit (geste) donc plus fort, avec } \\
\text { un vibrato plus lâche (il joue) }\end{array}$ \\
\hline 12 '00 & $\begin{array}{l}\text { Sth : Reprends depuis là et tu fais qu'est-ce } \\
\text { que tu préfères, surprise pour moi. }\end{array}$ \\
\hline
\end{tabular}

Ainsi, l'enseignant en formation sollicite puis prend en compte des souhaits de l'élève (11'00, 12'00) et les infléchit en fonction de conseils sur le jeu instrumental, distillés ici sous une forme très estompée (7'35, 9'10), mais qui demande à l'élève de faire des choix parmi plusieurs possibles dévoilés.

Pour conclure, l'enseignant en formation témoigne d'une conception de l'apprentissage technique qui prend son sens dans les possibles de l'interprétation d'une œuvre, mais en privilégiant amplement une dimension personnelle des choix du musicien instrumentiste. 
Ce qui implique également une prise de risques. Comme le met en exergue la sentence adressée à l'élève dans L3, lorsqu'elle termine de jouer un morceau :

Extrait 15' : Articulation technique-interprétation comme choix personnel

$14 ' 54$ Sth : Lopération est réussie mais le patient est mort!

Cette sentence, relativement opaque, rend paradoxalement manifeste ce qui semble être la limite de l'approche de l'interprétation en termes de « choix personnels » bien moins que comme compréhension de l'œuvre. Cela rend manifeste que si la mobilisation des conseils techniques fournis par l'enseignant durant la leçon constitue une des conditions nécessaires au jeu musical d'une pièce, elle doit être complétée par un apport du musicien-interprète. En revanche, bien que cet apport soit utilisé dans le contrat didactique, il n'est pas utilisable en tant qu'objet d'enseignement.

\section{CONCLUSIONS : L'ENSEIGNEMENT DE L'INTERPRÉTATION, UN OBJET D'ENSEIGNEMENT METTANT À L'ÉPREUVE DES OUTILS DIDACTIQUES ET LEURS USAGES}

Nous avons vu que l'apprentissage d'un instrument de musique présente le double enjeu de la maîtrise de l'instrument et du rapport à la musique que l'on peut produire, notamment à partir des œuvres écrites pour ce dernier. Avec les élèves débutants, cela se traduit le plus souvent, d'une part, par une articulation entre des contenus techniques visant la maîtrise du système corps-instrument et celle des écritures musicales; d'autre part, par des contenus interprétatifs instaurant un rapport sensible à la production sonore et à l'exécution des œuvres. Cette tension est propre au champ des pratiques musicales, comme le montrent les travaux sur les stratégies de travail des instrumentistes experts, et qui se constitue également en un véritable problème professionnel pour les enseignants. Ne pouvant pas «tout enseigner en même temps ", nous avons vu que les enseignants sont obligés de discriminer différents types de savoirs relatifs à cette problématique, à les organiser dans le temps de l'enseignement en fonction des contraintes que pose leur apprentissage, à les articuler en vue de construire des objets d'enseignement de plus en plus complexes, également en prenant en compte des capacités de l'élève.

Face à la problématique très complexe de l'enseignement de l'interprétation instrumentale auprès des élèves débutants, nous avons choisi dans cette étude d'analyser la manière dont ces objets d'enseignement complexes sont abordés par les enseignants en formation en termes de choix didactiques. Les résultats montrent des contrastes quant à l'usage, comme outil pour organiser leur enseignement, de l'une ou l'autre des deux conceptions « technique PUIS interprétation » et « interprétation ET techniques afférentes». Nos analyses montrent que ces conceptions constituent davantage un outil professionnel (Samurçay \& Pastré, 2004) qu'un script méthodique auquel les enseignants devraient se conformer. Certes, l'adhésion à l'une ou l'autre des conceptions oriente la lecture des situations d'enseignement et détermine, en partie, les choix didactiques. En même temps, à partir de ces " grilles de lecture », les enseignants doivent « savoir les utiliser » pour adapter leur enseignement en fonction des spécificités des contextes.

La prépondérance de la conception « technique PUIS interprétation » dans le corpus large de l'étude et dans celui restreint choisi pour ce texte n'est pas étonnante dans la mesure où elle préside à la structuration de la forme scolaire canonique "échauffement-exercices-jeu de l'œuvre », cela tant au niveau de la leçon que de l'organisation de l'année académique. Les données quantitatives montrent que les profils d'enseignement, sur le laps de temps important d'un semestre académique, produisent des résultats numériques très proches pour les deux cas de violoncelle (60\% MT contre $20 \% \mathrm{MI}$ ), qui sont significatifs des tendances générales adoptées par ces enseignants. Bien que certaines données montrent une proximité de surface entre l'enseignement de violoncelle 1B et de Harpe 1A, notamment dans les histogrammes, une distribution équilibrée des moments d'émergence des MI sur la plupart des leçons et les chiffres moyens d'occurrences MT/MI sur l'ensemble du semestre montrent clairement que l'enseignante de harpe mobilise une conception différente (avec des valeurs MT et MI très équilibrées et proches du $40 \%$ ).

Les histogrammes de Violoncelle 1A (Fig. 2) et de Violoncelle 1B (Fig. 4) de même que les données qualitatives montrent que les deux cas diffèrent sur 
plusieurs points, notamment par rapport à l'articulation des MT aux MI et aux autres savoirs, ainsi qu'aux moments d'émergence des occurrences MI dans la leçon. Ce constat rend manifeste la différence entre l'outil et son usage. Par rapport à ce point, nous pouvons avancer l'hypothèse que la différence d'expérience entre les enseignants en formation Ste (Violoncelle 1A) et Sth (Violoncelle 1B), respectivement en $1^{\text {re }}$ année et $2^{\mathrm{e}}$ année de master, constitue sans doute un facteur important permettant d'expliquer la variabilité de leurs conduites, malgré le fait d'adhérer dans l'ensemble à la même conception des rapports technique-interprétation. Ici encore, la relation des données quantitatives et qualitatives offre des pistes d'analyse intéressantes : alors que leurs pourcentages moyens d'occurrences sont très proches, et que les deux enseignants en formation progressent sur l'ensemble du semestre ${ }^{13}$, l'analyse qualitative de Violoncelle 1B montre que Sth a une gestion beaucoup plus efficace de la structuration des leçons (rappel systématique des devoirs à la maison $(\mathrm{MH})$ en début de leçon, davantage d'équilibre entre le temps consacré aux trois parties de la structure canonique) et plus de clarté dans l'articulation des apports MT/MI : que ce soient la dimension sensible et esthétique de la production sonore ou parfois l'œuvre musicale, ces éléments sont clairement placés comme un cadre justifiant les apports MT, plus nombreux, dans la leçon. Chez Ste (Violoncelle 1A, $1^{\text {ère }}$ année de master), l'articulation MT/MI est plus difficile et les analyses montrent qu'elle suspend très souvent l'enseignement des MI, pour privilégier des apports techniques de la leçon.

Les chiffres obtenus pour les deux cas de violoncelle versus celui de la harpe rendent donc manifeste le fait que les enseignants font appel à différents schémas d'organisation ayant une incidence dans les choix des contenus ainsi que leur ordonnancement et leurs stratégies de gestion, notamment en fonction des capacités des élèves. Par rapport à nos deux questions de recherche, avec les nuances qu'amènent les données en termes de progression de l'agir professionnel, l'on peut clairement constater des contrastes importants en ce qui concerne la distribution et l'articulation des contenus, la définition des milieux didactiques de travail et la co-construction des topoï des élèves.

Nous pouvons donc conclure que concernant la définition, la distribution et l'articulation des savoirs dans les différentes leçons, la conception «inter- prétation ET techniques afférentes », lorsqu'elle est basée sur l'œuvre musicale comme milieu didactique, conduit vers une co-construction équilibrée et articulée de différents contenus relatifs à la production musicale. Le cas Harpe $1 \mathrm{~A}$ (Sti, $2^{\mathrm{e}}$ année) montre clairement que les occurrences des contenus MI et MT manifestent des valeurs très proches (42.2\% et $40.2 \%$ respectivement). Les objets enseignés semblent cohérents et équilibrés avec les dimensions de l'œuvre et de la performance musicale mises en évidence dans la problématique. Par ailleurs, la perspective adoptée par cette enseignante en formation montre que les savoirs relatifs à l'accès de la lecture de partitions (ML) se distribuent également de manière équilibrée dans la mesure où les contenus « techniques» de lecture solfégique s'articulent en permanence avec une analyse des aspects « variables et implicites " (Hastings, 2011) des œuvres, par exemple le passage des phrases au phrasé, dans L3 ( $c f$. extrait 8). Ainsi, même l'organisation du travail à la maison implique un travail soutenu de la dimension MI : dans les histogrammes, l'on constate très souvent que les occurrences ML sont associées aux MI et MT. Du point de vue des topoï, l'on constate également que celui des rapports musicien-œuvre est prioritairement travaillé comme moyen de construction d'une posture interprétative chez l'élève, notamment dans les phases « échauffement » et " exercices » de la structure tripartite des leçons. Cela conduit à une approche assez particulière de la deuxième phase dans laquelle les besoins interprétatifs de l'œuvre donnent un sens aux exercices autour des apports sur la technique instrumentale. Chez cette enseignante, la dernière phase de la structure tripartite est consacrée au travail sur le topos musicien-instrument prioritairement focalisé sur la dimension interprétative que constitue l'expressivité du geste musical (Blum, 2001).

Ces constats contrastent de manière importante avec les productions enseignantes de la conception « technique PUIS interprétation » à laquelle adhèrent les deux cas de violoncelle. L'on relève chez ceux-ci un rapport de $60 \%$ de MT contre $20 \%$ de MI, qui oriente les contenus enseignés surtout sur un objet enseigné « maîtrise de l'instrument ». Par rapport à celui-là, les aspects MI s'orientent notamment sur la construction d'une "belle sonorité » (cf. extrait 2 : «pense à remplir ton son toujours plus c'est ton oreille qui doit te dire quel geste tu dois avoir ») intimement liée à l'efficacité du geste technique; cela au 
point que durant le temps d'ajustement de ce dernier, l'élève doit accepter des résultats sonores contraires à ses attentes ( $c f$. extrait 12 : «Appuie vraiment, sur le sol... ça va pas être joli, [plutôt] vraiment dégueulasse. »). Dans cette conception, le travail de l'œuvre devient souvent prétexte pour améliorer les acquis techniques, avec des conséquences importantes sur la construction de la dimension interprétative de la musique : d'un point de vue topogénétique, dans les deux cas l'on observe que souvent la responsabilité de l'interprétation est attribuée à l'élève mais sans faire de liens explicites avec les outils qui permettraient de la construire. Cette attribution concerne tant le topos musicien-instrument (cf. extrait 3: « profite de ce SOL à vide qui résonne »; $c f$. extrait 14 : « Là il y a des points sur les notes ... je te montre deux versions, laquelle tu préfères? $»)$, que celui musicienœuvre ( $c f$. extrait $15:$ "Reprends depuis là et tu fais qu'est-ce que tu préfères, surprise pour moi. »).

Le contraste entre les effets didactiques des deux conceptions sur une construction des savoirs musicaux dans les leçons se manifeste de manière assez tranchée en ce qui concerne les savoirs MI. L'analyse qualitative montre, en effet, une très forte tendance dans le cas 1 (Violoncelle 1A) et le cas 3 (Violoncelle 1B) à ne pas enseigner l'interprétatif, celui-ci étant, selon notre hypothèse, considéré comme un horizon lointain du statut de débutant des élèves. Ce trait explique que pour L3 du cas Violoncelle 1B, lorsque l'enseignant se retrouve face à une élève avancée, il s'autorise ce que l'on peut qualifier comme un enseignement de savoirs interprétatifs sur l'œuvre, qui laisse par ailleurs une large place à la sensibilité de l'élève. Mais selon nos observations, le plus souvent les jeunes enseignants qui s'inscrivent dans cette conception semblent très peu armés pour enseigner la dimension interprétative du jeu instrumental qui, dès lors, est dévolue à l'élève en termes non pas de savoirs mais d'une sensibilité esthétique qui semble difficilement « enseignable ». Cela semble poser un véritable problème aux enseignants en formation, que le deuxième cas (Harpe 1A) surmonte grâce à une très claire orientation sur l'œuvre musicale comme système sémiotique. Dans ce cas, l'interprétation semble bien constituer un « objet de savoirs » qui passe notamment par :

- le décodage des écritures concernées sur les partitions,

- une approche sémiotique de l'œuvre qui recourt principalement au fonctionnement de la « notation musicale », par exemple en descellant des structures peu évidentes (phrases, parties, phrasé et dialogues entre les deux instruments...)

- une approche herméneutique au sens d'Arbo (2000) qui, au-delà d'un simple décodage de la partition, est liée à une sensibilité construite du musicien-interprète relevant du rapport « général » du musicien à la sonorité instrumentale, en fonction d'une esthétique articulée à la musique comme produit historiquement et socialement situé.

Concernant le second aspect relatif aux conceptions comme outil professionnel, nous pouvons conclure que celle mobilisant l'œuvre comme milieu didactique prépondérant offre davantage de ressources pour la gestion de l'articulation techniqueinterprétation, l'enseignant pouvant largement s'appuyer sur les différents niveaux de lecture de l'œuvre pour articuler les savoirs MI avec les savoirs MT.

\section{NOTES}

1. Ce texte fait partie des travaux de diffusion des résultats de la recherche « LA PROFESSIONNALISATION DES ENSEIGNANTS EN FORMATION INITIALE. (Subside 100019-156730) financée par le Fonds national suisse pour la recherche scientifique (FNS).

2. This text is part of the work disseminating the results of a study on «THE PROFESSIONALIZATION OF TEACHERS IN INITIAL TEACHER TRAINING». (Grant 100019-156730) funded by the Swiss National Fund for scientific research (SNF).

3. Cette étude étant menée en Suisse romande, il convient de spécifier que les caractéristiques de l'enseignement musical dans les Hautes écoles de musique suivent un parcours semblable aux conservatoires français. En revanche, le Master en Pédagogie prévoit un nombre de crédits ETCS important pour la didactique de l'instrument. Dans ce cadre, les étudiants reçoivent une formation théorique qui est régulièrement évaluée au moment de donner des cours à leurs élèves devant leur formateur, pendant les deux années de formation.

4. À cet égard, concernant l'ambition de l'époque de tout pouvoir transcrire en notations musicales, l'on peut citer la crainte exprimée par Schubert « dans son Autobiographie: On paraît vouloir indiquer de nos jours, dans la musique, les nuances les plus fines; il en résulte une telle accumulation de termes techniques qu'on n'aperçoit plus les notes, tant il y a de signes musicaux. » (cité par Bosseur, 2005, p. 80). 
5. Dans cette optique, la possibilité très récente d'enregistrer une de ces performances n'y change rien, un enregistrement n'étant que la cristallisation d'une version de l'œuvre.

6. Ainsi, par exemple dans l'Elégie de Fauré, édition Peters, $\mathrm{n}^{\mathrm{o}} 7571$ datant de 1883, l'indication métronomique de 69 à la croche (rajoutée par l'éditeur, en s'inspirant de la version de 1901 pour violoncelle et orchestre) ne peut habituellement pas faire l'objet d'une interprétation littérale et entièrement partagée du caractère « adagio » de ce morceau.

7. C. Bellu, La transmission aux enfants débutants des savoirs dits "interprétatifs » en cours de violoncelle, thèse de doctorat en cours sous la direction d'Isabelle Mili, Université de Genève.

8. Relativement à notre corpus d'analyse, par exemple, le fait que les leçons aient été filmées durant le deuxième semestre de l'année scolaire explique, en partie, l'importance progressive que les enseignants débutants donnent à la dimension « interprétation de l'œuvre » vers la fin du semestre, la structure tripartite mentionnée impliquant pour la plupart des cas une performance publique des élèves dans le cadre de concerts et présentations de fin d'année.

9. Le corpus total de ce projet concerne 4 Hautes écoles de musique réparties sur le territoire suisse, impliquant 11 formateurs de didactique dans différents domaines instrumentaux et du chant, 11 enseignants en formation et 11 élèves.

10. Sur tous les histogrammes, la durée des leçons est indiquée en minutes; les occurrences des savoirs retenus s'expriment en nombre, et selon l'ordre d'apparition par minute retenue.

11. Cette caractéristique est commune à une très grande majorité des leçons (5) pour les 11 cas du corpus principal de la recherche et pas seulement aux trois cas choisis pour l'analyse dans ce texte.

12. Issue de la Méthode du jeune violoncelliste, de Feuillard. 13. Nous utilisons la diminution du nombre moyen d'occurrences de définitions des quatre types de savoirs par leçon comme un indice de progression, notamment dans le sens d'une gestion plus efficace du geste de définition et de la mobilisation d'autres gestes enseignants dans la gestion du rapport à l'élève (dévolutions, régulations).

\section{RÉFÉRENCES}

Arbo, A. (2000). Herméneutique de la musique? International Review of the Aesthetics and Sociology of Music, 31(1), 53-66.

Baily, J. (2001). Linteraction homme-instrument. Vers une conceptualisation. Cahiers d'ethnomusicologie
14 | 2001. [En ligne : http://journals.openedition.org/ ethnomusicologie/1835]

Blum, L. (2001). Geste instrumental et transmission musicale. Cahiers de musiques traditionnelles, 14, 237-248.

Boissière, A. (2011). André Schaeffner et les origines corporelles de l'instrument de musique. Methodos, 11 [En ligne : http://methodos.revues.org/2481\#quotation].

Bosseur, J.-Y. (2005). Du son au signe. Histoire de la notation musicale. Paris : Editions Alternatives.

Brousseau, G. (1991). Théorie des situations didactiques. Grenoble: La Pensée sauvage.

Cahiers d'ethnomusicologie, 2 (1989). Dossier Instrumental [En ligne : http://ethnomusicologie.revues.org/2323].

Da Lage, E., \& Vandiedonck, D. (2001). Impression d'originalité : «les hommes ressemblent plus à leur temps qu'à leur père ». Études de communication. Langages, information, médiation. Numéro spécial : Linterprétation : entre élucidation et création, 24. [En ligne http://edc.revues.org/1006].

Dogantan-Dack, M. (2012). The art of research in live music performance. Music Performance Reseach, 5, 34-48.

Feuillard, L.-R. (1970). La technique du violoncelle. Volume 1. Nice: Delrieu.

Feuillard, L.-R. (1953). Méthode du jeune violoncelliste. Paris : Delrieu

Goasdoué, R. (2004). Évolution des stratégies d'élaboration du projet musical et de sa réalisation dans l'apprentissage $d u$ violon. Thèse de doctorat en psychologie. Paris : Université de Paris-Nanterre.

Harnoncourt, N. (1982). Le Discours musical. Paris : Éditions Gallimard.

Hastings, C. (2011). How expert pianists interpret scores: A hermeneutical model of learning. International Symposium on Performance Science [En ligne http:// www.performancescience.org/ISPS2011/Proceedings/ Rows/062Hastings.pdf] (PDF).

Héroux, I., \& Fortier, M.-S. (2014). Expérimentation d'une nouvelle méthodologie pour expliciter le processus de création d'une interprétation musicale. Les cahiers de la Société québécoise de recherche en musique, 15(1), 67-79.

Imberty, M. (1998). De l'objectif au subjectif : points de repère pour une réflexion sur l'interprétation dans l'analyse et dans le travail musicien. Revue belge de Musicologie, 52, 215-233.

Imberty, M. (1997). Formes de la répétition et formes des affects du temps dans l'expression musicale. Musica Scientice, 1(1), 33-63.

Mauss, M. (1960). Sociologie et anthropologie. Paris : PUF

Nattiez, J.-J. (1987). Musicologie générale et sémiologie. Paris : Christian Bourgois.

Rastier, F. (2001). Eléments de théorie des genres. Texto! [En ligne, consulté en juin 2012 : http://www.revuetexto.net/Inedits/Rastier/Rastier_Elements.html].

Ravet, H. (2005). Linterprétation musicale comme performance : interrogations croisées de musicologie et de sociologie. Musurgia, 4(12), Analyser l'interpréta- 
tion (2005), 5-18. Paris : ESKA [En ligne, consulté le 18.03.2016 : http://www.jstor.org/stable/40591408].

Rickenmann, R., \& De la Calle, L. (2015). Construction sociale des émotions esthétiques : genre d'œuvre et fonctions dans la perezhivanie. Communication dans le cadre du Colloque Vygotski 2015 (Paris-CNAM) et ICME4 (Genève-Université de Genève).

Samurçay, R., \& Pastré, P. (Eds.) (2004). Recherches en didactique professionnelle. Toulouse : Octarès.

Schubauer-Leoni, M.-L., \& Leutenegger, F. (2002). Expliquer et comprendre dans une approche clinique / expérimentale du didactique ordinaire. Dans F. Leutenegger \& M. Saada-Robert (Eds.) (2002), Expliquer et comprendre en sciences de l'éducation (p. 227-251). Bruxelles: De Boeck.

Schütz, A. (2006). Faire de la musique ensemble. Une étude des rapports sociaux. Sociétés, 93(3),15-28.

Sensevy, G., \& Mercier, A. (dir.) (2007). Agir Ensemble. L'action didactique conjointe du professeur et des élèves. Rennes: PUR.

Sensevy, G. (2006). Laction didactique. Eléments de théorisation. Schweizerische Zeitschrift für Bildungswissenschaften, 28(2), 205-225.

Sensevy, G., Mercier, A., \& Schubauer-Leoni, M.-L. (2000). Vers un modèle de l'action didactique du professeur. À propos de la course à 20. Recherches en Didactique des Mathématiques, 20(3), 263-304.

Sève, B (2011). Linstrument de musique : présentation du numéro. Methodos, 11 [En ligne : https://methodos. revues.org/2599\#quotation].

Vincent, G. (1996). Pratiques culturelles ou formes symboliques? Hermès, La Revue, 20(2), 155-162.

Willener, A (1997). Les instrumentistes d'orchestres symphoniques. Variations diaboliques. Paris : L'Harmattan. 